Pacific Journal of Mathematics

ON THE BIRMAN INVARIANTS OF HEEGAARD SPLITTINGS 


\title{
ON THE BIRMAN INVARIANTS OF HEEGAARD SPLITTINGS
}

\author{
José María Montesinos ANd CARmen SAFOnT
}

\author{
Dedicated to Professor Joan S. Birman
}

\begin{abstract}
As Professor Birman indicated in [Bil] the homological information about a given Heegaard splitting of genus $g$ is contained in a double coset in the group of symplectic $2 g \times 2 g$ integer matrices with respect to a suitable subgroup. She found in [Bi1] a determinant invariant of this double coset and we prove in this paper that that invariant (strengthened a bit when the first torsion number is even) is complete. We obtain this result by characterizing the double coset in terms of the linking form of the manifold lifted to a handlebody of the Heegaard splitting and by finding complete invariants of this lifted form. Professor Birman has kindly pointed out to us that the characterization of the double cosets by means of her invariant is contained in the unpublished manuscript [Bi-J].
\end{abstract}

1. Introduction. Let $F$ be an orientable, closed surface embedded in a (closed, orientable) 3-manifold $M$. The pair $(M, F)$ is said to be a Heegaard splitting if $F$ separates $M$ in two handlebodies. If the genus of $F$ is $g$, then $(M, F)$ is a Heegaard splitting of genus $g$. Two Heegaard splittings $(M, F)$ and $\left(M^{\prime}, F^{\prime}\right)$, are equivalent if $(M, F) \cong\left(M^{\prime}, F^{\prime}\right)$, i.e. if there exists a homeomorphism $h: M \rightarrow M^{\prime}$ such that $h(F)=F^{\prime}$.

Every closed, orientable 3-manifold has Heegaard splittings as was remarked by Heegaard. A considerable effort has been made in the past to achieve the classification of Heegaard splittings. Waldhausen [Wa] proved that any two genus $g$ Heegaard splittings of the 3-sphere $S^{3}$ are equivalent (ambient isotopic, indeed). He used the classical result of Reidemester and Singer that two Heegaard splittings of the same manifold are stably equivalent, i.e. they are ambient isotopic after adding enough trivial handles to both Heegaard splittings. Bonahon-Otal [Bo-O] classified the Heegaard splittings of the lens spaces and they showed that there is exactly one for each genus. This is not true in general: Engmann [Eng] gave the first example of a manifold (a connected sum of lenses) with two inequivalent Heegaard splittings of genus 2 (for a different proof and generalization see [Bi1]). Later Birman-González-Montesinos [BGAM] distinguished two Heegaard splittings of genus two of an irreducible manifold (a homology sphere), using geometric methods introduced earlier 
by Birman-Hilden [BiH] (see [Mo2]). The first numerical invariants of Heegaard splittings were introduced by Birman in her 1975 paper [Bi1]. Her invariants were used to provide new proofs of Engmann's results and were useful for finding many examples of inequivalent Heegaard splittings and of inequivalent plats (see [Mo1], [Mo2], for instance).

The starting point of Birman was the observation of the fact that there is a one to one correspondence between equivalence classes of (oriented) Heegaard splittings of genus $g$ and double cosets in the mapping class group of the closed orientable surface $F_{g}$ with respect to the subgroup of automorphisms of $F_{g}$ extending to a handlebody bounded by $F_{g}$. She then applied the homology functor to this problem and considered double cosets in the group of symplectic $2 \times 2$ integer matrices with respect to a suitable subgroup. All homology information about a given Heegaard splitting is contained in the corresponding double coset. Invariants of this double coset are invariants of the Heegaard splitting. She found easily a determinant invariant, but it seemed hard to prove that this was a complete invariant of the double coset or to find what was missed by this determinant. A great deal of work was done in this direction in [Bi1].

On the other hand, we found out that the linking form of the manifold lifted to one of the handlebodies separated by a Heegaard surface provides an invariant of the Heegaard splitting in question [S]. Our invariant was also a determinant, and we soon realized that it was related to Birman's invariant. In this paper, which completes [MS], we prove that the congruence class of the linking form associated to a Heegaard splitting and the double coset contain exactly the same information. We are very grateful to Joan Birman for pointing out to us that the lifted linking form and the double coset associated to a Heegaard splitting were more closely related than we thought, and for informing us that complete invariants of double cosets were found independently in an unpublished joint work by her and Dennis Johnson ([Bi-J]), which she facilitated to us.

For us the lifted linking form associated to a Heegaard splitting, which is defined on a free abelian group, has been the main object of attention. Invariants of the congruence class of this linking are invariants of the Heegaard splitting. We have solved completely the problem of classifying the congruence classes. This problem reduces to finding complete invariants of a matrix of the linking form of the manifold under congruence by unimodular matrices of certain type. Our solution is based on the classification of linking forms on finite abelian groups, done in 
[Se] for groups without 2-torsion, and in $[\mathbf{B u}]$, [W] (see also [K]) for 2-groups. A complete invariant of the congruence class is the determinant of a matrix obtained from the linking form matrix, mod $\tau_{1}$ (sometimes $\bmod 2 \tau_{1}$, when $\tau_{1}$ is even) where $\tau_{1}$ is the first torsion coefficient of the group $H_{1}(M ; \mathbf{Z})$.

We show that our determinant invariant in most cases coincides with Birman's invariant in [Bi1]. Finally, we prove that the lifted linking form, which depends on the double coset, indeed determines it. Hence, by means of the linking form, we have found complete invariants of the double coset. It turns out to be that these invariants coincide with those found by Birman-Johnson [Bi-J].

Not many new applications can be expected, but we give as a new result the classification of the Heegaard splittings of the connected sum of two lens spaces $L(p, s), L\left(p^{\prime}, s^{\prime}\right)$ when $p=p^{\prime}$.

Part of this work was contained in the Ph.D. Thesis [S] of the second author, written under the direction of the first one. We want to thank Craig Hodgson for very interesting discussions which were very helpful to bring this paper to an end, and Joan Birman for useful information.

2. Linking form associated to Heegaard splittings. Let $\vec{M}$ be a closed, oriented three-manifold and let $(\vec{M}, X)$ be an oriented Heegaard splitting (i.e. $X$ and $\overline{M-X}$ are handlebodies) we say that $(\vec{M}, X)$ and $\left(\vec{M}^{\prime}, X^{\prime}\right)$ are equivalent if there is an (orientation-preserving) homeomorphism $f: \vec{M} \rightarrow \vec{M}^{\prime}$ mapping $X$ to $X^{\prime}$. We are interested in invariants of the equivalence class of the Heegaard splitting $(\vec{M}, X)$. In this paper, these will be the invariants of the linking form of $\vec{M}$ lifted to $X$.

Let $T(M)$ be the torsion subgroup of $H_{1}(M, \mathbf{Z})$, and $T(X)$ the preimage of $T(M)$ under the inclusion induced homomorphism

$$
j_{*}: H_{1}(X, \mathbf{Z}) \rightarrow H_{1}(M ; \mathbf{Z})
$$

We define

$$
\begin{gathered}
\mathscr{L}(\vec{M}, X): T(X) \times T(X) \rightarrow \mathbf{Q} / \mathbf{Z}, \\
(x, y) \mapsto \mathscr{L}\left(j_{*} x, j_{*} y\right)
\end{gathered}
$$

REMARK 1. The nondegenerate bilinear füıul associated to $\mathscr{L}(\vec{M}, X)$ is the linking form $\mathscr{L}$ of $\vec{M}$. For, let $\operatorname{Nul} \mathscr{L}(\vec{M}, X)$ be the set of $x \in T(X)$ such that $\mathscr{L}(\vec{M}, X)(x, y)=0$ for every $y \in T(X)$. Since $j_{*}$ is 
onto and $\mathscr{L}$ is nondegenerate, this is equivalent to $j_{*} x=0$. Thus

$$
\operatorname{Nul} \mathscr{L}(\vec{M}, X)=\operatorname{Ker}\left(j_{*}: T(X) \rightarrow T(M)\right) .
$$

Assume that $\tau_{1}, \tau_{2}, \ldots, \tau_{r}$ are the torsion coefficients of $H_{1}(M ; \mathbf{Z})$, where $\tau_{1}\left|\tau_{2} \cdots\right| \tau_{r}$. Select a basis $B=\left(a_{1}, \ldots, a_{s}\right)$ of $T(X)$ such that

$$
\left(\tau_{1} a_{1}, \ldots, \tau_{r} a_{r}, a_{r+1}, \ldots, a_{s}\right)
$$

is a basis of $\operatorname{Ker} j_{*}$. A matrix $A(X, B)$ of $\mathscr{L}(\vec{M}, X)$ with respect to the basis $B$ has the form

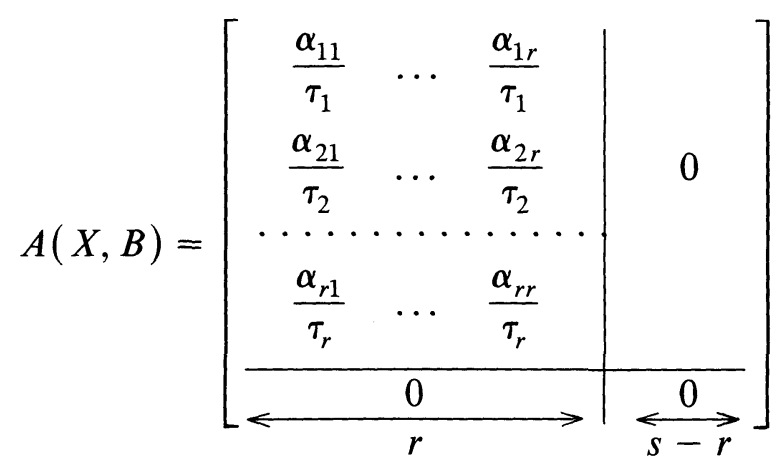

Denote by $\hat{A}(X, B)$ the matrix $\left(\alpha_{l j}\right)$ defined from $A(X, B)$ only up to sum of

$$
\left[\begin{array}{lll}
\tau_{1} & & \\
& \ddots & \\
& & \tau_{r}
\end{array}\right] Z,
$$

where $Z$ denotes any $r \times r$ integer matrix. Then

$$
\left[\begin{array}{lll}
\frac{1}{\tau_{1}} & & \\
& \ddots & \\
& & \frac{1}{\tau_{r}}
\end{array}\right] \hat{A}(X, B)
$$

is a matrix of the linking form $\mathscr{L}$ of $\vec{M}$ with respect to the basis $\left(\bar{a}_{1}, \ldots, \bar{a}_{r}\right)$ of $T(M)$, where $\bar{a}_{i}=j_{*}\left(a_{i}\right), i=1, \ldots, r$.

We say that $\mathscr{L}(\vec{M}, X)$ and $\mathscr{L}\left(\vec{M}^{\prime}, X^{\prime}\right)$ are congruent if there exists some isomorphism $h: T(X) \rightarrow T\left(X^{\prime}\right)$ such that

$$
\mathscr{L}\left(\vec{M}^{\prime}, X^{\prime}\right)(h x, h y)=\mathscr{L}(\vec{M}, X)(x, y) \quad \text { for } x, y \in T(X) .
$$

It follows from Remark 1 that $h$ projects to an isomorphism $\bar{h}: T(M) \rightarrow$ $T\left(M^{\prime}\right)$ which preserves the linking forms $\mathscr{L}, \mathscr{L}^{\prime}$. Therefore the coordinate 
matrix $C \in \mathrm{GL}(s, \mathbf{Z})$ of $h$ with respect to bases $B, B^{\prime}$ selected as above has the following form since the orders of the torsion elements of $T(M)$ are preserved by $\bar{h}$ :

$$
C=\left[\begin{array}{cccc|c}
c_{11} & \frac{\tau_{2}}{\tau_{1}} c_{11} & \ldots & \frac{\tau_{r}}{\tau_{1}} c_{1 r} & * \\
c_{21} & c_{22} & \ldots & \frac{\tau_{r}}{\tau_{2}} c_{2 r} & * \\
\ldots \ldots \ldots & \ldots \ldots \ldots \ldots \ldots & \\
c_{r 1} & & \ldots & c_{r r} & * \\
\hline * & * & \ldots & * & *
\end{array}\right]
$$

The rows of $C$ are the coordinates of the images of the elements of $B$, with respect to $B^{\prime}$. Moreover, $C$ satisfies

$$
C A\left(X^{\prime}, B^{\prime}\right) C^{t}=A(X, B) \text { on } \mathbf{Q} / \mathbf{Z} \text {. }
$$

Let $\hat{C}$ be the submatrix of $C$ formed by the first $r$ rows and columns. Then

$$
\hat{C}\left[\begin{array}{ccc}
\frac{1}{\tau_{1}} & & \\
& \ddots & \\
& & \frac{1}{\tau_{r}}
\end{array}\right] \hat{A}\left(X^{\prime}, B^{\prime}\right) \hat{C}^{t}=\left[\begin{array}{ccc}
\frac{1}{\tau_{1}} & & \\
& \ddots & \\
& & \frac{1}{\tau_{r}}
\end{array}\right] \hat{A}(X, B)
$$

on $\mathbf{Q} / \mathbf{Z}$.

Think of $A(X, B)$ as a matrix with entries in the cyclic group of $r$ elements, which we denote $\left(\tau_{r}\right)$, naturally embedded in $\mathbf{Q} / \mathbf{Z}$.

Let $\mathrm{SL}^{ \pm}\left(s,\left(\tau_{r}\right)\right)$ denote the group of $s \times s$ matrices with entries in $\left(\tau_{r}\right)$ with determinant $\pm 1 \bmod \tau_{r}$. It is well-known that the natural homomorphism

$$
\mathrm{GL}(s, \mathbf{Z}) \rightarrow \mathrm{SL}^{ \pm}\left(s,\left(\tau_{r}\right)\right)
$$

is onto (see [Bass] page 8, Lemma 1.1), so for any matrix

$$
C \in \mathrm{SL}^{ \pm}\left(s,\left(\tau_{r}\right)\right)
$$

there exists $C^{\prime} \in \mathrm{GL}(s, \mathbf{Z})$ such that $C=C^{\prime}+\tau_{r} Z$, where $Z$ is an integer matrix. And $C$ satisfies (2) and (3) if and only if $C^{\prime}$ does. Thus, congruence of $A(X, B)$ under matrices of $\mathrm{GL}(s, \mathbf{Z})$ reduces to congruence under matrices of $\mathrm{SL}^{ \pm}\left(s,\left(\tau_{r}\right)\right)$.

If $(\vec{M}, X)$ and $\left(\vec{M}^{\prime}, X^{\prime}\right)$ are equivalent oriented Heegaard splittings, then $\mathscr{L}(\vec{M}, X)$ and $\mathscr{L}\left(\vec{M}^{\prime}, X^{\prime}\right)$ are congruent. The congruence class of 
$\mathscr{L}(\vec{M}, X)$ is associated to the equivalence class of $(\vec{M}, X)$ and it follows the

THEOREM 2.1. The congruence class of $A(X, B)$, thought of as a matrix with entries in the cyclic group $\left(\tau_{r}\right)$, under matrices of $\mathrm{SL}^{ \pm}\left(s,\left(\tau_{r}\right)\right)$ of the form (2) determines the congruence class of $\mathscr{L}(\vec{M}, X)$, and therefore is an invariant of the equivalence class of $(\vec{M}, X)$.

3. Classifying the linking $\mathscr{L}(\vec{M}, X)$. Our goal is to give a complete set of invariants of the congruence class of $\mathscr{L}(\vec{M}, X)$. The first invariant is given in the following theorem (remember that $r$ is the number of torsion coefficients, and that $s=\operatorname{genus}(X)-\operatorname{rank} H_{1}(M ; \mathbf{Z})$ ).

3.1. TheOREM. If $r=s$, det $\hat{A}(X, B)\left(\bmod \tau_{1}\right)$ is an invariant of the congruence class of $\mathscr{L}(\vec{M}, X)$.

Proof. First of all note that since $A(X, B)$ is a matrix with entries in $\mathbf{Q} / \mathbf{Z}$, det $\hat{A}(X, B)$ is only defined $\bmod \tau_{1}$. Let $C \in \mathrm{GL}(r, \mathbf{Z})$ be a matrix of the form

$$
C=\left[\begin{array}{cccc}
c_{11} & \frac{\tau_{2}}{\tau_{1}} c_{12} & \ldots & \frac{\tau_{r}}{\tau_{1}} c_{1 r} \\
c_{21} & c_{22} & \ldots & \frac{\tau_{r}}{\tau_{2}} c_{2 r} \\
\ldots \ldots \ldots & \ldots \ldots & \ldots \\
c_{r 1} & c_{r 2} & \cdots & c_{r r}
\end{array}\right]
$$

Thus

$$
A\left(X^{\prime}, B^{\prime}\right)=C A(X, B) C^{t}=C\left[\begin{array}{lll}
\frac{1}{\tau_{1}} & & \\
& \ddots & \\
& & \frac{1}{\tau_{r}}
\end{array}\right] \hat{A}(X, B) C^{t} .
$$

We will see that

$$
C\left[\begin{array}{ccc}
\frac{1}{\tau_{1}} & & \\
& \ddots & \\
& & \frac{1}{\tau_{r}}
\end{array}\right]=\left[\begin{array}{lll}
\frac{1}{\tau_{1}} & & \\
& \ddots & \\
& & \frac{1}{\tau_{r}}
\end{array}\right] C^{\prime}
$$


for a matrix $C^{\prime}$ with $\operatorname{det} C^{\prime}=\operatorname{det} C$. In fact, we have

$$
\begin{aligned}
& {\left[\begin{array}{cccc}
c_{11} & \frac{\tau_{2}}{\tau_{1}} c_{12} & \ldots & \frac{\tau_{r}}{\tau_{1}} c_{1 r} \\
c_{21} & c_{22} & \ldots & \frac{\tau_{r}}{\tau_{2}} c_{2 r} \\
\ldots & \ldots & \ldots & \ldots \ldots \\
c_{r 1} & c_{r 2} & \ldots & c_{r r}
\end{array}\right]\left[\begin{array}{cccc}
\frac{1}{\tau_{1}} & & & \\
& \frac{1}{\tau_{2}} & & \\
& & \ddots & \\
& & & \frac{1}{\tau_{r}}
\end{array}\right]}
\end{aligned}
$$

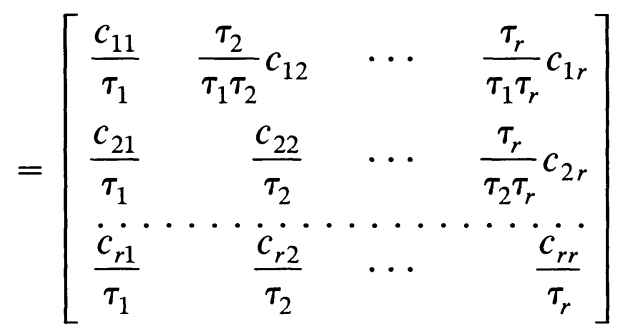

$$
\begin{aligned}
& =\left[\begin{array}{cccc}
\frac{1}{\tau_{1}} & & & \\
& \frac{1}{\tau_{2}} & & \\
& & \ddots & \\
& & & \frac{1}{\tau_{r}}
\end{array}\right]\left[\begin{array}{cccc}
c_{11} & c_{12} & \ldots & c_{1 r} \\
\frac{\tau_{2}}{\tau_{1}} c_{21} & c_{22} & \ldots & c_{2 r} \\
\ldots & \ldots & \ldots & \ldots \\
\frac{\tau_{r}}{\tau_{1}} c_{r 1} & \frac{\tau_{r}}{\tau_{2}} c_{r 2} & \ldots & c_{r r}
\end{array}\right]
\end{aligned}
$$

Let $C=\left(d_{i j}\right)$ and let $C^{\prime}=\left(d_{l j}^{\prime}\right)$ be the matrix on the right in the last term. Then

$$
d_{i j}^{\prime}=\frac{\tau_{i}}{\tau_{j}} d_{i j}
$$

and clearly $\operatorname{det} C^{\prime}=\operatorname{det} C$. Thus $\hat{A}\left(X^{\prime}, B^{\prime}\right)=C \hat{A}(X, B) C^{t}$ and the theorem follows.

We will see later that $\operatorname{det} \hat{A}(X, B)\left(\bmod \tau_{1}\right)$ is precisely the invariant found by Joan Birman in [Bi1]. We also will see that it is a complete invariant when $\tau_{1}$ is odd, and almost a complete invariant when $\tau_{1}$ is even. To determine the complete invariants we first solve the problem for the case when $\tau_{1}$ is a prime power. In [Se], Seifert proved that the problem of classifying the linking form $\mathscr{L}$ of $\vec{M}$ reduces to classifying the restrictions of $\mathscr{L}$ to the $p$-primary summands of $T(M)$, since these are direct summands orthogonal with respect to the linking form $\mathscr{L}$. 
Our problem has an extra difficulty. For each prime divisor $p$ of $\tau_{r}$, let $T_{p}(M)$ be the $p$-primary summand of $T(M)$, and let $T_{p}(X)$ be the preimage of $T_{p}(M)$ under $j_{*}$. Define

$$
\mathscr{L}_{p}(\vec{M}, X)=\mathscr{L}(\vec{M}, X) \mid T_{p}(X) \times T_{p}(X)
$$

The groups $\operatorname{Ker} j_{*} \leq T_{p}(X) \leq T(X)$ are all of them free abelian of rank $s$, hence $T(X)$ is not the direct sum of the subgroups $T_{p}(X)$.

It is clear that if $\mathscr{L}(\vec{M}, X)$ and $\mathscr{L}\left(\vec{M}^{\prime}, X^{\prime}\right)$ are congruent, then $\mathscr{L}_{p}(\vec{M}, X)$ and $\mathscr{L}_{p}\left(\vec{M}^{\prime}, X^{\prime}\right)$ are congruent too. For an isomorphism $h$ : $T(X) \rightarrow T\left(X^{\prime}\right)$ preserving $\mathscr{L}(\vec{M}, X), \mathscr{L}\left(\vec{M}^{\prime}, X^{\prime}\right)$ restricts to an isomorphism $h_{p}: T_{p}(X) \rightarrow T_{p}\left(X^{\prime}\right)$ preserving $\mathscr{L}_{p}(\vec{M}, X), \mathscr{L}_{p}\left(\vec{M}^{\prime}, X^{\prime}\right)$. The converse is not so clear, since given a sequence of linking-preserving isomorphisms $h_{p}: T_{p}(X) \rightarrow T_{p}\left(X^{\prime}\right)$ we cannot, in principle, extend them to an isomorphism between $T(X)$ and $T\left(X^{\prime}\right)$. What we will do is, roughly, to extend each $h_{p}$ and then compose the extensions.

Let $B=\left(a_{1}, \ldots, a_{s}\right)$ be a basis of $T(X)$ chosen as in $\$ 2$. If $\tau_{1}=$ $p^{e_{1}} d_{1}, \ldots, \tau_{r}=p^{e_{r}} d_{r}$, where $0 \leq e_{1} \leq \cdots \leq e_{r}$ and $d_{i} \neq \equiv \bmod p$, then

$$
B_{p}=\left(d_{1} a_{1}, \ldots, d_{r} a_{r}, a_{r+1}, \ldots, a_{s}\right)
$$

is a basis of $T_{p}(X)$ and

$$
A_{p}\left(X, B_{p}\right)=\left[\begin{array}{ccc|c}
\frac{d_{1} \alpha_{11}}{p^{e_{1}}} & \ldots & \frac{d_{r} \alpha_{1 r}}{p^{e_{1}}} & \\
\frac{d_{1} \alpha_{21}}{p^{e_{2}}} & \ldots & \frac{d_{r} \alpha_{2 r}}{p^{e_{2}}} & 0 \\
\frac{d_{1} \alpha_{r 1}}{p^{e_{r}}} & \ldots & \frac{d_{r} \alpha_{r r}}{p^{e_{r}}} & \\
\hline \underset{S-r}{\stackrel{0}{\longrightarrow}} & 0 & \stackrel{0}{\longrightarrow}
\end{array}\right]
$$

is a matrix of $\mathscr{L}_{p}(\vec{M}, X)$ with respect to $B_{p}$. Let $\hat{A}_{p}\left(X, B_{p}\right)=\left(d_{j} \alpha_{i j}\right)$.

Note that the discussion prior to the statement of Theorem 2.1 applies to $\mathscr{L}_{p}(\vec{M}, X)$ as well as to $\mathscr{L}(\vec{M}, X)$, and it follows that $\mathscr{L}_{p}(\vec{M}, X)$ and $\mathscr{L}_{p}\left(\vec{M}^{\prime}, X^{\prime}\right)$ are congruent if there exists a matrix $C_{p}$ in $\mathrm{GL}(s, \mathbf{Z})$ or, equivalently, in $\mathrm{SL}^{ \pm}\left(s,\left(p^{e_{r}}\right)\right)$ such that

$$
C_{p} A_{p}\left(X, B_{p}\right) C_{p}^{t}=A_{p}\left(X^{\prime}, B_{p}^{\prime}\right) \text { on } \mathbf{Q} / \mathbf{Z}
$$


The matrix $C_{p}$ satisfying (5) has the form

(6)

$$
C_{p}=\left[\begin{array}{cccc|c}
c_{11} & p^{e_{2}-e_{1}} c_{12} & \ldots & p^{e_{r}-e_{1}} c_{1 r} & * \\
c_{21} & c_{22} & \ldots & p^{e_{r}-e_{2}} c_{2 r} & * \\
\ldots \ldots & \ldots \ldots & \ldots & \ldots \ldots \ldots & \\
c_{r 1} & c_{r 2} & \ldots & c_{r r} & * \\
\hline * & * & \ldots & * & * \\
\stackrel{r}{\longleftrightarrow}
\end{array}\right]
$$

We first determine a system of generators of the subgroup $G_{s}$ of $\mathrm{SL}^{ \pm}\left(s,\left(p^{e_{s}}\right)\right)$ of the form (6) (compare [Se]). Consider matrices of $G_{s}$ of the form

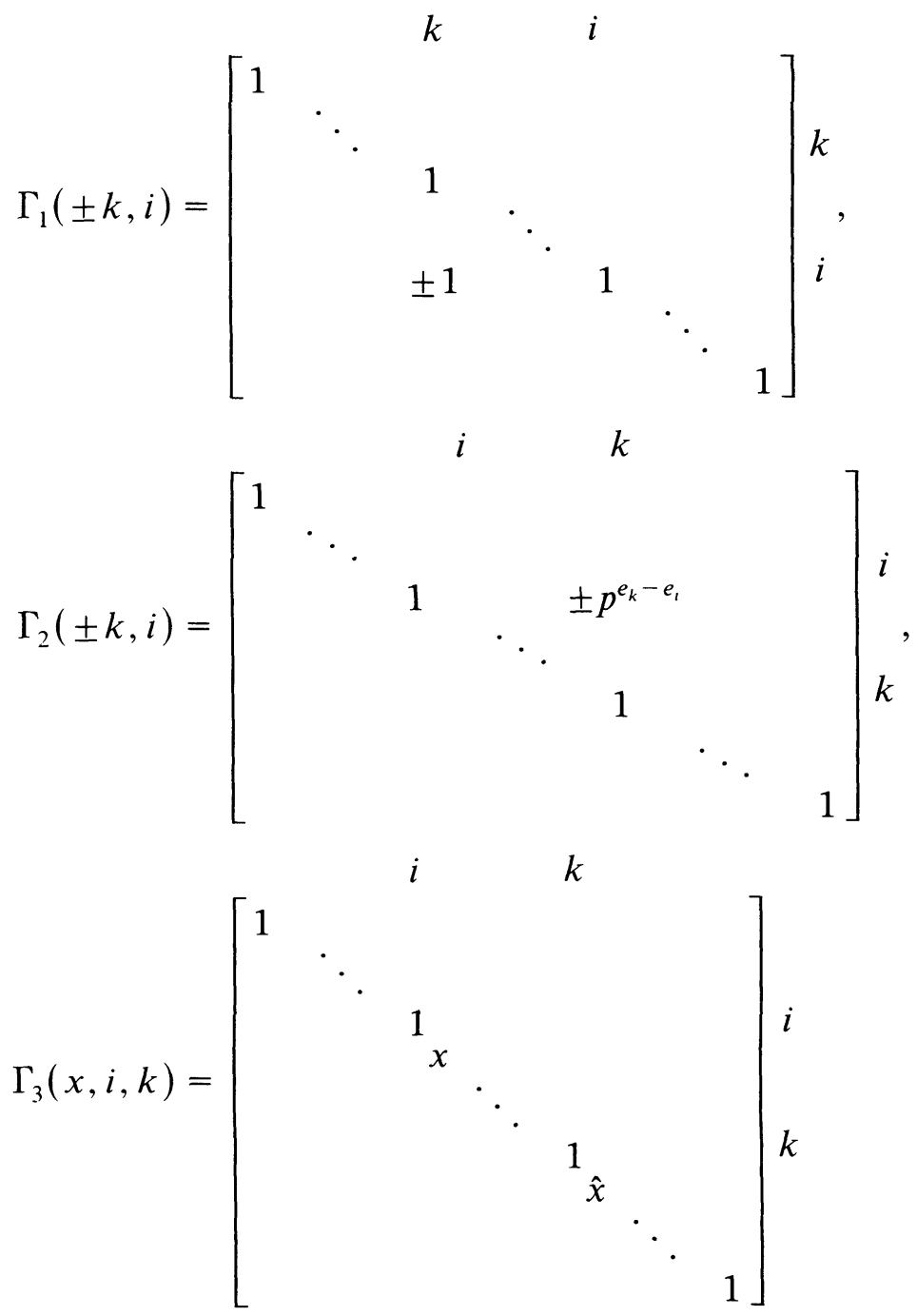


where $x \not \equiv 0 \bmod p$ and $x \hat{x} \equiv \pm 1 \bmod p^{e_{r}}$. This set of matrices generates $G_{s}$. We prove this as in [Se]. Given $C \in G_{s}$, the first row must contain $c_{1 i} \not \equiv 0 \bmod p$, otherwise $\operatorname{det} C \equiv 0 \bmod p$. If $c_{11} \equiv 0 \bmod p$, we take $C \Gamma_{1}(1, i)$. The effect on $C$ of right multiplication by $\Gamma_{1}(1, i)$ is to add the $i$ th column to the first. Therefore we can assume $c_{11} \not \equiv 0 \bmod p$. Multiplying to the right by $\Gamma_{3}\left(\hat{c}_{11}, 1, s\right)$ we can assume $c_{11} \equiv 1 \bmod p^{e_{r}}$. The element $c_{1 k}$ is of the form $c_{1 k}^{\prime} p^{e_{k}-e_{1}}$. We multiply our matrix to the right by $\Gamma_{2}(-k, 1) c_{1 k}^{\prime}$ times. The effect is to subtract the first column multiplied by $c_{1 k}^{\prime} p^{e_{k}-e_{1}}$ from the $k$ th column. Thus we can assume that $c_{1 k} \equiv 0$ $\bmod p^{e_{r}}, k>1$. The result of deleting the first row and column of $C$ is a $(s-1) \times(s-1)$ matrix of $G_{s-1}$. By induction we can assume that $c_{i j} \equiv 0 \bmod p^{e_{r}}$, for $j>i$, and that $c_{i i} \equiv 1 \bmod p^{e_{r}}$. We can use $\Gamma_{3}(-1, s, 1)$ to make $c_{s s} \equiv 1 \bmod p^{e_{r}}$ also. We now multiply $C$ by $\Gamma_{1}(-k, s)$ to the left $c_{k s}$ times, thus killing $c_{k s}$, and continue this way with the remaining rows, starting from the last, until $C$ is converted in the identity matrix. This proves our claim.

Assume now that for every prime divisor $p$ of $\tau_{r}$, the forms $\mathscr{L}_{p}\left(\vec{M}^{\prime}, X\right)$ and $\mathscr{L}_{p}\left(\vec{M}^{\prime}, X^{\prime}\right)$ are congruent. For a fixed $p$, suppose that $C_{p}$ is a matrix of $\operatorname{SL}^{ \pm}\left(s,\left(p^{e_{r}}\right)\right)$ satisfying (6). Then $C_{p} \in G_{s}$ and therefore can be expressed as a product of matrices of type $\Gamma_{1}( \pm k, i), \Gamma_{2}( \pm k, i), \Gamma_{3}(x, k, i)$. Since $\tau_{r}=p^{e_{r}} d_{r}$ where $d_{r} \not \equiv 0 \bmod p$, an equality of the form

$$
1=\hat{p} p^{e_{r}}+\hat{d} d_{r}
$$

can be found, for some integers $\hat{p}, \hat{d}$. Then, for $j=1, \ldots, s$,

$$
a_{j}=\hat{p} p^{e_{r}} a_{j}+\hat{d} \frac{d_{r}}{d_{j}}\left(d_{j} a_{j}\right)
$$

Let $\hat{d}_{j}=\hat{d}_{r} / d_{j}$.

To the matrix $\Gamma_{1}( \pm k, i)$ we can associate the following matrix

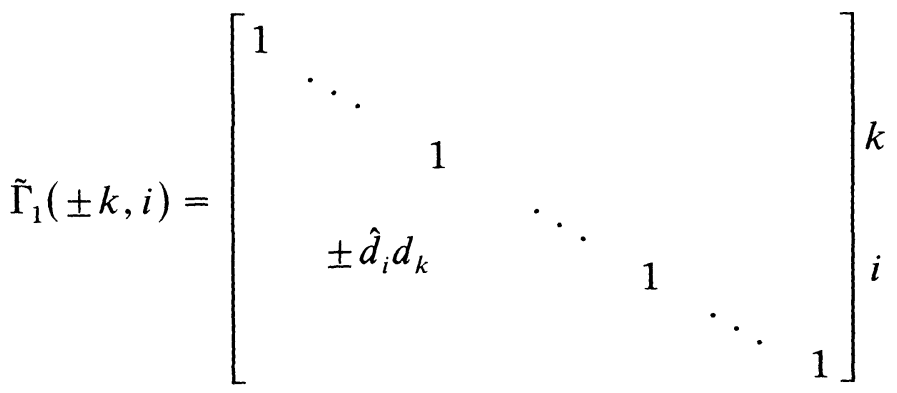


If $\Gamma_{1}( \pm k, i)$ is thought of as the reduction $\bmod p^{e_{r}}$ of the coordinate matrix of an automorphism of $T_{p}(X)$ with respect to the basis $B_{p}=$ $\left(d_{1} a_{1}, \ldots, d_{r} a_{r}, a_{r+1}, \ldots, a_{s}\right)$, then $\tilde{\Gamma}_{1}( \pm k, i)$ can be thought of as the reduction $\bmod \tau_{r}$ of the coordinate matrix with respect to

$$
B=\left(a_{1}, \ldots, a_{r}, a_{r+1}, \ldots, a_{s}\right)
$$

of an automorphism of $T(X)$ which induces the identity on $\oplus_{q \neq p} T_{q}(M)$ and which on $T_{p}(M)$ induces the same automorphism as $\Gamma_{1}( \pm k, i)$.

The matrix associated the same way to $\Gamma_{2}( \pm k, i)$ is

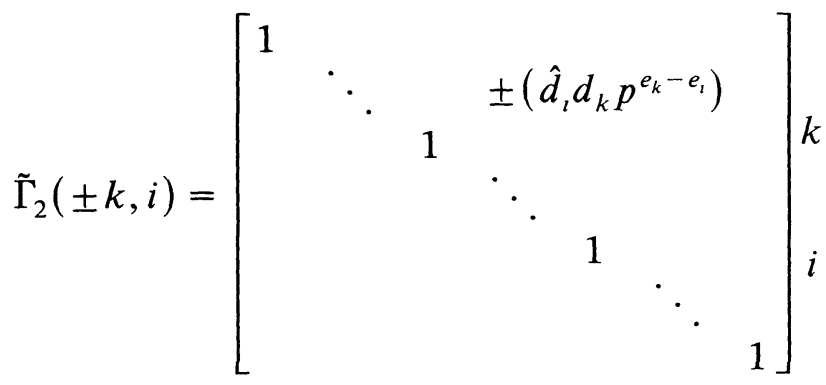

$$
\begin{aligned}
& i \quad k
\end{aligned}
$$

and the matrix associated to $\Gamma_{3}(x, k, i)$ is

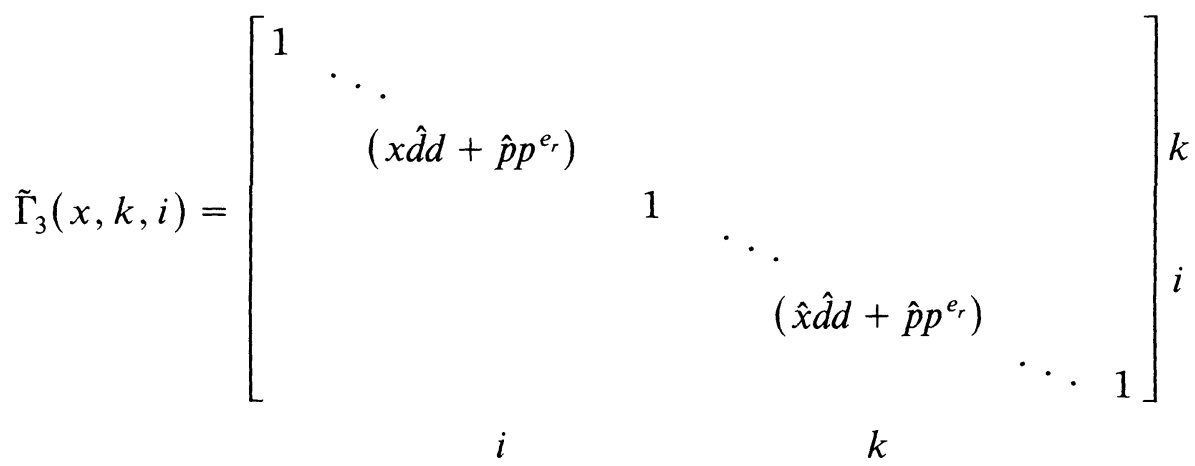

(for $x \not \equiv 0 \bmod p, \hat{x} x \equiv 1 \bmod p^{e_{r}}$ ).

The matrices $\tilde{\Gamma}_{i}, i=1,2,3$ so defined have determinant $\pm 1 \bmod \tau_{r}$. Replacing the matrix $\Gamma_{i}$ by its associate $\tilde{\Gamma}_{l}$ in the expression of $C_{p}$, we get a matrix $\tilde{C}_{p}$ with determinant $\pm 1 \bmod \tau_{r}$, which induces on $T(M)$ an automorphism which is the identity on the complement of $T_{p}(M)$ and which on $T_{p}(M)$ induces the same automorphism as $C_{p}$ (which preserves the linking $\left.\mathscr{L}_{p}=\mathscr{L} \mid T_{p}(M) \times T_{p}(M)\right)$. Finally, the product $C$ of such matrices $\tilde{C}_{p}$, when $p$ ranges over all the prime divisors of $\tau_{r}$ is a matrix of determinant $\pm 1 \bmod \tau_{r}$ which preserves $\mathscr{L}$ and thus satisfies (3). Thus we have the following. 
3.2. THEOREM. Let $(\vec{M}, X),\left(\vec{M}^{\prime}, X^{\prime}\right)$ be oriented Heegaard splittings. Then $\mathscr{L}(\vec{M}, X)$ and $\mathscr{L}\left(\vec{M}^{\prime}, X^{\prime}\right)$ are congruent if and only if for every prime divisor $p$ of $\tau_{r} \mathscr{L}_{p}(\vec{M}, X)$ and $\mathscr{L}_{p}\left(\vec{M}^{\prime}, X^{\prime}\right)$ are congruent.

4. Complete congruence invariants of $\mathscr{L}_{p}(\vec{M}, X)$. In this section we fix a prime $p$ and investigate invariants of the congruence class of $\mathscr{L}_{p}(\vec{M}, X)$, or equivalently, invariants of the matrix $A_{p}\left(X, B_{p}\right)$ given in $\S 3$ under congruence by matrices of the group $G_{s}$. We solve the problem, giving a complete invariant of this congruence class.

First we assume that $T_{p}(M)$ is isomorphic to

$$
\left(p^{e_{1}}\right)^{m_{1}} \oplus \cdots \oplus\left(p^{e_{t}}\right)^{m_{t}}, \quad 0<e_{1}<\cdots<e_{t}
$$

where $\left(p^{e_{t}}\right)^{m_{i}}$ is the direct sum of $m_{i}$ copies of the cyclic group of $p^{e_{t}}$ elements, and take a basis $\left(b_{1}, \ldots, b_{s}\right)$ of $T_{p}(X)$ such that

$$
\left(p^{e_{1}} b_{1}, \ldots, p^{e_{1}} b_{m_{1}}, p^{e_{2}} b_{m_{1}+1}, \ldots, p^{e_{t}} b_{m}, b_{m+1}, \ldots, b_{s}\right)
$$

is a basis of $\operatorname{Ker} j_{*}$, where $m=m_{1}+\cdots+m_{t}$. This may require a reordering in the basis $B_{p}$ given in $\S 3$ and of the matrix $A_{p}\left(X, B_{p}\right)$ but we keep denoting the new basis and matrix the same way. In this section we

\begin{tabular}{|c|c|c|c|c|}
\hline$\frac{A_{11}}{p^{e_{1}}}$ & $\frac{A_{12}}{p^{e_{1}}}$ & $\cdots$ & $\frac{A_{1 t}}{p^{e_{1}}}$ & 0 \\
\hline$\frac{A_{21}}{p^{e_{2}}}$ & $\frac{A_{22}}{p^{e_{2}}}$ & . & $\frac{A_{2 t}}{p^{e_{2}}}$ & 0 \\
\hline$\cdots$ & $\cdots$ & $\cdots$ & $\ldots$ & $\cdots$ \\
\hline$\frac{A_{t 1}}{p^{e_{t}}}$ & $\frac{A_{t 2}}{p^{e_{t}}}$ & $\cdots$ & $\frac{A_{t t}}{p^{e_{t}}}$ & 0 \\
\hline 0 & 0 & 0 & 0 & 0 \\
\hline
\end{tabular}
write the matrix $A_{p}\left(X, B_{p}\right)$ as follows:

where $A_{i}$ are integer matrices. Then $\hat{A}_{p}\left(X, B_{p}\right)=\left(A_{i j}\right)$.

4.1. THEOREM. det $\hat{A}_{p}\left(X, B_{p}\right) \bmod p^{e_{1}}$ is an invariant of the congruence class of $\mathscr{L}_{p}(\vec{M}, X)$ if $m=s$. If $m<s$, then any two matrices $A_{p}\left(X, B_{p}\right)$ and $A_{p}\left(X^{\prime}, B_{p}^{\prime}\right)$ of $\mathscr{L}_{p}(\vec{M}, X), \mathscr{L}_{p}\left(\vec{M}^{\prime}, X^{\prime}\right)$ are congruent by some $C \in G_{s}$ provided that $\mathscr{L}_{p}, \mathscr{L}_{p}^{\prime}$ are congruent. 
Proof. The first part of the theorem is a particular case of Theorem 3.1 .

For the last part of the theorem, notice that the first $m \times m$ blocks of $A_{p}\left(X, B_{p}\right), A_{p}\left(X^{\prime}, B_{p}^{\prime}\right)$ are congruent via a matrix $C^{\prime}$ such that $\operatorname{det} C^{\prime} \not \equiv 0$ $\bmod p$, because they represent the linking forms $\mathscr{L}_{p}, \mathscr{L}_{p}^{\prime}$. If $s>m$ we can enlarge $C^{\prime}$ to an $s \times s$ matrix $C$ having determinant $\pm 1 \bmod p^{e_{r}}$.

4.2. Corollary. When $r<s$, any two forms $\mathscr{L}(\vec{M}, X)$ and $\mathscr{L}\left(\vec{M}^{\prime}, X^{\prime}\right)$ are congruent, provided that $\mathscr{L}$ and $\mathscr{L}^{\prime}$ are congruent.

Proof. It follows from Theorems 4.1 and 3.2.

REMARK. Remember that $s=\operatorname{genus}(X)-\operatorname{rank} H_{1}(M)$ and that $r$ is the number of torsion coefficients. We have genus $(X) \geq \operatorname{rank} H_{1}(M)+r$. Thus, by Corollary 4.2, if the Heegaard splitting does not have minimum genus, we cannot obtain any invariant of $(\vec{M}, X)$ by means of $\mathscr{L}(\vec{M}, X)$.

If the matrix $A_{11}$, which is symmetric mod $p^{e_{1}}$, is normalized so that it becomes symmetric mod $p^{e_{1}+1}$ we denote $\left(A_{i j}\right)$ by $\hat{A}_{p}^{s}\left(X, B_{p}\right)$.

4.3. THEOREM. If $p=2$ and $m=s$, $\operatorname{det} \hat{A}_{p}^{s}\left(X, B_{p}\right) \bmod 2^{e_{1}+1}$ is an invariant of the congruence class $\left\{C A_{p}\left(X, B_{p}\right) C^{t}, C \in G_{s}\right\}$ (and, therefore, an invariant of $\left.\mathscr{L}_{p}(\vec{M}, X)\right)$ if:

(i) the diagonal elements of $A_{11}$ are $\equiv 0 \bmod 2$,

(ii) $A_{11}$ has even order.

Remarks. Property (i) is preserved under congruence (note that $A_{11}$ gives an even quadratic form). Property (ii) is a consequence of property (i) (see the proof of Theorem 4.4).

Proof. Under congruence $A_{11}$ remains symmetric. Therefore the proof of the theorem will be a consequence of that of Theorem 3.1 once we show that $\operatorname{det} \hat{A}_{p}^{s}\left(X, B_{p}\right)$ is well defined $\bmod 2^{e_{1}+1}$. If we replace the diagonal element $\alpha$ of $A_{11}$ by $\alpha+x 2^{e_{1}}$ we change the determinant of $\hat{A}_{p}^{s}\left(X, B_{p}\right)$ by $x 2^{e_{1}}(\mid$ Adjoint $\alpha \mid)$. But the matrix (Adjoint $\alpha$ ) $\bmod 2$ has determinant zero. In fact (Adjoint $\alpha$ ) mod 2 is upper-diagonal in blocks and the first block is $A_{11}$ mod 2 in which we have deleted the row and column containing $\alpha$. But this deleted $A_{11}$ mod 2 is symplectic of odd order and its determinant must vanish. If we replace a non-diagonal $\alpha_{i j} \in A_{11}$ by $\alpha_{i j}+x 2^{e_{1}}$ we must 
replace its symmetric $\alpha_{j i}=\alpha_{i j}$ by $\alpha_{j i}+x 2^{e_{1}}$, to maintain $A_{11}$ symmetric. The det $\hat{A}_{p}^{s}\left(X, B_{p}\right)$ changes by $x 2^{e_{1}}\left(\mid\right.$ Adjoint $\alpha_{i j}|+|$ Adjoint $\left.\alpha_{j i} \mid+\dot{2}\right)$. But $\bmod 2$ Adjoint $\alpha_{i j}$ and Adjoint $\alpha_{j i}$ have the same determinant, therefore $\operatorname{det} \hat{A}_{p}^{s}\left(X, B_{p}\right)$ does not change $\bmod 2^{e_{1}+1}$. If we replace $\alpha \in A_{1 i}, i>1$, by $\alpha+x 2^{e_{1}}$, then $\operatorname{det} \hat{A}_{p}^{s}\left(X, B_{p}\right)$ changes by $x 2^{e_{1}}(\mid$ Adjoint $\alpha \mid)$. But Adjoint $\alpha \bmod 2$ is the result of deleting in an upperdiagonal block matrix a row and a column passing through different diagonal blocks; the resulting determinant is zero.

Suppose that $m=s$ and we are given matrices $A_{p}\left(X, B_{p}\right), A_{p}\left(X^{\prime}, B_{p}^{\prime}\right)$ corresponding to $(\vec{M}, X),\left(\vec{M}^{\prime}, X^{\prime}\right)$ such that $\mathscr{L}_{p}$ and $\mathscr{L}_{p}^{\prime}$ are congruent. Since $A_{p}\left(X, B_{p}\right) A_{p}^{\prime}\left(X^{\prime}, B_{p}^{\prime}\right)$ represent the linking forms $\mathscr{L}_{p}, \mathscr{L}_{p}^{\prime}$, it follows from [Se], [Bu], [W] (see [K]) that we can find matrices $C, C^{\prime}$ of the form (6) with $\operatorname{det} C \not \equiv O(p), \operatorname{det} C^{\prime} \not \equiv O(p)$ and such that

$$
\begin{aligned}
& C A_{p}\left(X, B_{p}\right) C^{t}=N, \\
& C^{\prime} A_{p}^{\prime}\left(X^{\prime}, B_{p}^{\prime}\right) C^{\prime t}=N,
\end{aligned}
$$

where $N$ is a normal form for the linking form of the manifold. The matrix $N$ is diagonal in blocks of the following type (generating blocks):

Case 1. $p$ odd. The blocks are $\left[1 / p^{k}\right]$ and $\left[n / p^{k}\right]$ where $n$ is a fixed quadratic non-residue mod $p$ (the smallest one, for instance).

Case 2. $p=2$. The blocks are

$$
\left[\frac{1}{2^{k}}\right], \quad\left[\begin{array}{cc}
0 & \frac{1}{2^{k}} \\
\frac{1}{2^{k}} & 0
\end{array}\right], \quad k \geq 1 ;\left[\begin{array}{cc}
\frac{1}{2^{k-1}} & \frac{1}{2^{k}} \\
\frac{1}{2^{k}} & \frac{1}{2^{k-1}}
\end{array}\right], \quad\left[\frac{-1}{2^{k}}\right], \quad k \geq 2,
$$

and $\left[5 / 2^{k}\right],\left[-5 / 2^{k}\right], k \geq 3$. The blocks in $N$ are ordered so that the blocks corresponding to the same power of $p$ go together, and here the $(1 \times 1)$-blocks go first. In our case the first block is one of the above with $k=e_{1}$.

We now take the diagonal matrix $D_{x}=\left(d_{i j}\right)$ such that $d_{i j}=0, i \neq j$; $d_{i i}=1, i>i$; and $d_{11}=x$ is such that $x \cdot \operatorname{det} C \equiv 1 \bmod p^{e_{r}}$. Define $D^{\prime}$ similarly. Thus we have that $D C=\tilde{C}$ and $D^{\prime} C^{\prime}=\tilde{C}^{\prime}$ have determinant 1 $\bmod p^{e_{r}}$ and

$$
\begin{aligned}
& \tilde{C} A_{p}\left(X, B_{p}\right) \tilde{C}^{t}=S, \\
& \tilde{C}^{\prime} A_{p}^{\prime}\left(X^{\prime}, B_{p}^{\prime}\right) \tilde{C}^{\prime t}=S ;
\end{aligned}
$$


where $S, S^{\prime}$ differ from $N$ in the first block. For $S$ this has one of the following forms

$$
\begin{aligned}
{\left[\frac{x^{2}}{p^{e_{1}}}\right], \quad\left[\frac{n x^{2}}{p^{e_{1}}}\right], \quad\left[\frac{x^{2}}{2^{e_{1}}}\right], \quad\left[\begin{array}{cc}
0 & \frac{x}{2^{e_{1}}} \\
\frac{x}{2^{e_{1}}} & 0
\end{array}\right], \quad \text { if } e_{1} \geq 1, } \\
{\left[\begin{array}{cc}
\frac{x^{2}}{2^{e_{1}-1}} & \frac{x}{2^{e_{1}}} \\
\frac{x}{2^{e_{1}}} & \frac{1}{2^{e_{1}-1}}
\end{array}\right], \quad\left[\frac{-x^{2}}{2^{e_{1}}}\right], \quad \text { if } e_{1} \geq 2, \quad \text { or }\left[\frac{ \pm 5 x^{2}}{2^{e_{1}}}\right] }
\end{aligned}
$$

if $e_{1} \geq 3$, and similarly for $S^{\prime}$ replacing $x^{\prime}$ by $x$.

Assume $\operatorname{det} \hat{A}_{p}\left(X, B_{p}\right) \equiv \operatorname{det} \hat{A}_{p}^{\prime}\left(X^{\prime}, B_{p}^{\prime}\right) \bmod p^{e_{1}}$. Then, by Theorem 4.1, we obtain $\operatorname{det} \hat{S} \equiv \operatorname{det} \hat{S}^{\prime} \bmod p^{e_{1}}$ and since the determinants corresponding to the generating blocks are $\not \equiv 0 \bmod p$, we conclude $x^{2} \equiv x^{\prime 2}$ $\bmod p^{e_{1}}$. We distinguish two cases

Case 1. $p$ odd. Then $S$ and $S^{\prime}$ coincide and $\operatorname{det} \hat{A_{p}}\left(X, B_{p}\right)$ determines the congruence class of $A_{p}\left(X, B_{p}\right)$ by elements of $G_{s}$.

\section{Case 2. p even.}

Case 2.1. $e_{1} \leq 2$. In this case $x^{2} \equiv x^{\prime 2} \bmod 2^{e_{1}}$ implies $x \equiv \pm x^{\prime}$ $\bmod 2^{e_{1}}$. If $x \equiv x^{\prime} \bmod 2^{e_{1}}$ then $S=S^{\prime}$. If $x \equiv-x^{\prime} \bmod 2^{e_{1}}$ we take $-D$ instead of $D$, and we get also $S=S^{\prime}$. Therefore also in this case det $\hat{A}_{p}\left(X, B_{p}\right)$ is a complete invariant for the congruence class of $A_{p}\left(X, B_{p}\right)$ by elements of $G_{s}$.

Case 2.2. $e_{1} \geq 3$. In this case $x$ is congruent $\bmod 2^{e_{1}}$ to $\pm x^{\prime}$ or to $\pm\left(1+2^{e_{1}-1}\right) x$. These four cases are reduced to two as in Case 2.1. In this case if the first block of $S$ is a $(1 \times 1)$-block then $S=S^{\prime}$, and $\operatorname{det} \hat{A}_{p}\left(X, B_{p}\right)$ determines the congruence class of $A_{p}\left(X, B_{p}\right)$. If the first block is a $(2 \times 2)$-block this is not quite so, and there are in general two congruence classes $\left\{A_{p}\left(X, B_{p}\right)\right\},\left\{A_{p}^{\prime}\left(X^{\prime}, B_{p}^{\prime}\right)\right\}$ such that

$$
\operatorname{det} \hat{A}_{p}\left(X, B_{p}\right) \equiv \operatorname{det} A_{p}^{\prime}\left(X^{\prime}, B_{p}^{\prime}\right) \bmod 2^{e_{1}}
$$

as the next example shows. 
EXAMPLE. Consider the matrices

$$
A_{p}\left(X, B_{p}\right)=\left[\begin{array}{cc}
0 & \frac{1}{8} \\
\frac{1}{8} & 0
\end{array}\right], \quad A_{p}^{\prime}\left(X^{\prime}, B_{p}^{\prime}\right)=\left[\begin{array}{cc}
0 & \frac{5}{8} \\
\frac{5}{8} & 0
\end{array}\right]
$$

then there is no $C=\left[\begin{array}{ll}a & b \\ c & d\end{array}\right]$ with $a d-b c \equiv \pm 1 \bmod 8$ and such that $C A_{p}\left(X, B_{p}\right) C^{t}=A_{p}^{\prime}\left(X^{\prime}, B_{p}^{\prime}\right)$ in $\mathbf{Q} / \mathbf{Z}$. We will distinguish them as follows.

In Case 2.2 the matrices $A_{p}\left(X, B_{p}\right), A_{p}^{\prime}\left(X^{\prime}, B_{p}^{\prime}\right)$ satisfy the conditions of Theorem 3.2 hence $\operatorname{det} \hat{A}_{p}^{s}\left(X, B_{p}\right)$, $\operatorname{det} \hat{A}_{p}^{\prime s}\left(X^{\prime}, B_{p}^{\prime}\right) \bmod 2^{e_{1}+1}$ are congruence-invariants. Assume these two coincide. Then $x^{2} \equiv x^{\prime 2} \bmod 2^{e_{1}+1}$. Therefore $x \equiv \pm x^{\prime}$ or $\pm\left(1+2^{e_{1}}\right) x^{\prime} \bmod 2^{e_{1}+1}$ which implies $x \equiv \pm x^{\prime}$ $\bmod 2^{e_{1}}$. Thus we have:

4.4. ThEOREM. $\operatorname{det} \hat{A_{p}}\left(X, B_{p}\right) \bmod 2^{e_{1}}$ determines completely the congruence class $\left\{C A_{p}\left(X, B_{p}\right) C^{t}, C \in G_{s}\right\}$ if one of the following holds

(i) $p$ odd,

(ii) $p=2, e_{1} \leq 2$,

(iii) $p=2, e_{1} \geq 3$ and there exists a diagonal element $a_{l i}$ of $A_{11}$ such that $a_{1 i} \not \equiv 0 \bmod 2$.

Otherwise (i.e. if $p=2, e_{1} \geq 3$ and $a_{i i} \equiv 0 \bmod 2$, for all $a_{i i}$ in $A_{11}$ ) that congruence class is totally determined by $\operatorname{det} \hat{A}_{p}^{s}\left(X, B_{p}\right) \bmod 2^{e_{1}+1}$.

4.5. COROLlaRY. When $r=s$ (i.e. when the number of torsion coefficients equals genus $(X)$ - rank $\left.H_{1}(M)\right)$, the set

$\left\{\operatorname{det} \hat{A_{p}}\left(X, B_{p}\right)\left(\bmod p^{e(p)}\right) \mid p\right.$ is prime and $\left.\tau_{1}=p^{e(p)} d_{p}, d_{p} \not \equiv 0 \bmod p\right\}$

is a complete invariant of the congruence class of $\mathscr{L}(\vec{M}, X)$ unless 8 divides $\tau_{1}$ and $a_{i i} \equiv 0 \bmod 2$ for all diagonal elements $a_{i i}$ in the block $A_{11}$ of $A_{2}\left(X, B_{2}\right)$. In this case, the set

$\left\{\operatorname{det} \hat{A}_{p}\left(X, B_{p}\right) \bmod p^{e(p)} \mid p\right.$ is odd prime and $\tau_{1}=p^{e(p)} d_{p}$,

$$
\left.d_{p} \not \equiv 0 \bmod p\right\}
$$

$\cup\left\{\operatorname{det} \hat{A}_{2}^{s}\left(X, B_{2}\right) \bmod 2^{e+1}\right.$, if $\left.\tau_{1}=2^{e} \cdot d, d \not \equiv 0 \bmod 2\right\}$

is a complete invariant of the congruence class of $\mathscr{L}(\vec{M}, X)$.

5. Complete congruence invariants of $\mathscr{L}(\vec{M}, X)$. In this section we gather the results we have obtained through the preceding sections. Theorem 5.1 gives a complete invariant of the congruence class of 
$\mathscr{L}(\vec{M}, X)$, and therefore it gives all the invariants of the Heegaard splittings $(\vec{M}, X)$ that can be obtained using the methods of this paper.

Recall that, if rank $H_{1}(M, \mathbf{Z})=f$ and $T(M)$ is isomorphic to

$$
\left(\tau_{1}\right) \oplus\left(\tau_{2}\right) \oplus \cdots \oplus\left(\tau_{r}\right)
$$

where $\tau_{i} \mid \tau_{i+1}$ for $i=1, \ldots, r-1$ and $\left(\tau_{i}\right)$ denotes the cyclic group of order $\tau_{l}\left(\tau_{1}>1\right)$, then $M$ does not admit any Heegaard splitting of genus less than $f+r$.

5.1. TheOREM. Let $(\vec{M}, X)$ be an oriented Heegaard splitting of genus g. Then

(1) If $g>f+r$ we cannot obtain any invariant of $(\vec{M}, X)$ using $\mathscr{L}(\vec{M}, X)$.

(2) If $g=f+r$ and $\tau_{1}$ is the first torsion coefficient of $T(M)$ then

$$
\operatorname{det} \hat{A}(X, B) \bmod \tau_{1}
$$

is a complete invariant of the congruence class of $\mathscr{L}(\vec{M}, X)$ unless $\tau_{1}=$ $2^{e} u_{1}, u_{1} \not \equiv 0 \bmod 2, e \geq 3$ and whenever $\tau_{i}=2^{e} u_{\imath}, u_{i} \not \equiv 0 \bmod 2$, then $\alpha_{u} \equiv 0 \bmod 2$. In that case

$$
\operatorname{det} \hat{A}^{s}(X, B) \bmod 2 \tau_{1}
$$

is a complete invariant of $\mathscr{L}(\vec{M}, X)$, where $\hat{A}^{s}(X, B)$ is obtained normalizing $\hat{A}(X, B)$ so that

$$
\frac{\alpha_{l j}}{\tau_{i}} \equiv \frac{\alpha_{j i}}{\tau_{j}} \quad \bmod 2 \quad \text { for } 1 \leq i, j \leq k
$$

Proof. Part (1) is proved in Corollary 4.2. Part (2) follows from Corollary 4.5 if one notices that for each prime divisor $p$ of $\tau_{1}$, $\operatorname{det} \hat{A}_{p}\left(X, B_{p}\right)=d_{1} \cdots d_{r} \operatorname{det} \hat{A}(X, B)$, with the notation of $\S 3$. This follows from the form of $A_{p}\left(X, B_{p}\right)$ in (4). Finally, normalizing $\hat{A}_{p}\left(X, B_{p}\right)$ to $\hat{A}_{p}^{s}\left(X, B_{p}\right)$ for $p=2$ means to take $d_{j} \alpha_{i j} \equiv d_{i} \alpha_{j l} \bmod p^{e+1}$, for $1 \leq i$, $j \leq k$, i.e.

$$
\begin{gathered}
\frac{d_{J} \alpha_{i j}}{p^{e}} \equiv \frac{d_{i} \alpha_{j l}}{p^{e}} \bmod p, \\
\frac{d_{\imath} d_{j} \alpha_{i j}}{\tau_{i}} \equiv \frac{d_{i} d_{j} \alpha_{j l}}{\tau_{j}} \bmod p,
\end{gathered}
$$

which is equivalent to $\alpha_{i j} / \tau_{i} \equiv \alpha_{j l} / \tau_{j} \bmod p$ since $d_{i} \not \equiv 0 \not \equiv d_{j} \bmod p$. 
6. Birman's invariants. Birman approached the geometry of a Heegaard splitting looking at it as the union of two handlebodies glued together through a homeomorphism of their boundaries. Let us review her notation ([Bi1], [Bi2]).

Let $(\vec{M}, X)$ be an oriented Heegaard splitting. Let $X_{g}$ be an (oriented) standard handlebody of genus $g$, and let $\tau$ be a fixed orientationreversing automorphism of $X_{g}$. Let $u, v \tau: X_{g} \rightarrow \vec{M}$ be orientation-preserving embeddings of $X_{g}$ onto $X$ and $\overline{M-X}$, respectively. Then

$$
\vec{M} \cong\left(X_{g}\right)_{1} \cup_{\phi \tau}\left(X_{g}\right)_{2}:=\vec{M}_{\phi}
$$

where $\left(X_{g}\right)_{1}$ and $\left(X_{g}\right)_{2}$ are copies of $X_{g}$, and $\phi=u^{-1} \cdot v \mid$ is an orientation-preserving automorphism of $\partial X_{g}$ thought of as a homeomorphism from $\partial\left(X_{g}\right)_{1}$ to $\partial\left(X_{g}\right)_{2}$. The map $\phi$ is called a gluing map for $(\vec{M}, X)$. If $\sigma, \tau$ are orientation-preserving automorphisms of $\partial X_{g}$ extending to $X_{g}$ thus $\sigma \phi \tau$ is also a gluing map for the Heegaard splitting. Actually, as it was proven by Joan Birman in [Bil], there is a 1-1 correspondence between equivalence classes of oriented Heegaard splittings and double cosets in the mapping class group $\mathscr{M}_{g}$ of $\partial X_{g}$ modulo the subgroup $\mathscr{T}_{g}$ of (classes of) automorphisms of $\partial X_{g}$ which extend to $X_{g}$.

Now let

$$
\begin{aligned}
& \mathscr{M}_{g_{*}}=\left\{h: H_{1}\left(\partial X_{g} ; \mathbf{Z}\right) \rightarrow H_{1}\left(\partial X_{g} ; \mathbf{Z}\right): h=\phi_{*}, \phi \in \mathscr{M}_{g}\right\}, \\
& \mathscr{T}_{g_{*}}=\left\{h \in \mathscr{M}_{g_{*}}: h=\phi_{*}, \phi \in \mathscr{T}_{g}\right\} .
\end{aligned}
$$

Then the double coset $\mathscr{T}_{g_{*}} \phi_{*} \mathscr{T}_{g_{*}}$ is an invariant of the oriented Heegaard splitting $(\vec{M}, F)$.

As it was pointed out by Seifert [Se], matrices of the linking form $\mathscr{L}$ of $\vec{M}$ can be obtained from any Heegaard diagram of $\vec{M}$. By completing a Heegaard diagram to symplectic bases of the homology group of the Heegaard surface, the homology gluing map of the Heegaard splitting is expressed in terms of intersections of the curves of the extended Heegaard diagram, which suffice to compute $\mathscr{L}$. In particular it follows that the equivalence class of the linking form $\mathscr{L}(\vec{M}, X)$ defined in $\S 2$ depends on the double coset $\mathscr{T}_{g_{*}} \phi_{*} \mathscr{T}_{g_{*}}$ of a gluing map for $(\vec{M}, X)$. In $\S 8$ we will prove the converse: The congruence class of $\mathscr{L}(\vec{M}, X)$ determines the double coset $\mathscr{T}_{g_{*}} \phi_{*} \mathscr{T}_{g_{*}}$. It was known to Joan Birman that the linking form $\mathscr{L}$ of $\vec{M}$ determines what she called the stable double coset of any oriented Heegaard splitting of $\vec{M}$ (stable double cosets correspond to stable equivalence of Heegaard splittings. The Reidemeister-Singer Theorem states that any two Heegaard splittings of a given manifold are stably equivalent). This result is in unpublished lecture notes for the CBMS Conference at Blacksburg, Va., 1977 [Bi2]. 
Now we show that det $\hat{A}(X, B) \bmod \tau_{1}$ is precisely Birman's invariant given in [Bi1]. Birman extracted a determinant invariant of the double coset $\mathscr{T}_{g_{*}} \phi_{*} \mathscr{T}_{g_{*}}$ using a coordinate matrix of $\phi_{*}$ with respect to some symplectic matrix of $H_{1}\left(\partial X_{g}\right)$. Let $\varepsilon: H_{1}\left(\partial X_{g}\right) \rightarrow H_{1}\left(X_{g}\right)$ be the inclusion induced homomorphism. Starting with a symplectic basis

$$
\left(c_{1}, \ldots, c_{g}, a_{1}, \ldots, a_{g}\right)
$$

such that $\left(c_{1}, \ldots, c_{g}\right)$ is a basis of $\operatorname{Ker} \varepsilon$, and $\left(\bar{a}_{1}, \ldots, \bar{a}_{g}\right)$, where $\bar{a}_{i}=\varepsilon a_{l}$, $i=1 \cdots g$, is a basis of $H_{1}\left(X_{g}\right)$, with the property that $\left(\tau_{1} \bar{a}_{1}, \tau_{2} \bar{a}_{2}, \ldots, \tau_{r} \bar{a}_{r}, \bar{a}_{r+1}, \ldots, \bar{a}_{s}\right)$ is a basis for $\operatorname{Ker}\left(H_{1}\left(X_{g}\right) \rightarrow H_{1}\left(M_{\phi}\right)\right)$, the coordinate matrix of $\phi_{*}$ is of the form (notation of [Bi1]):
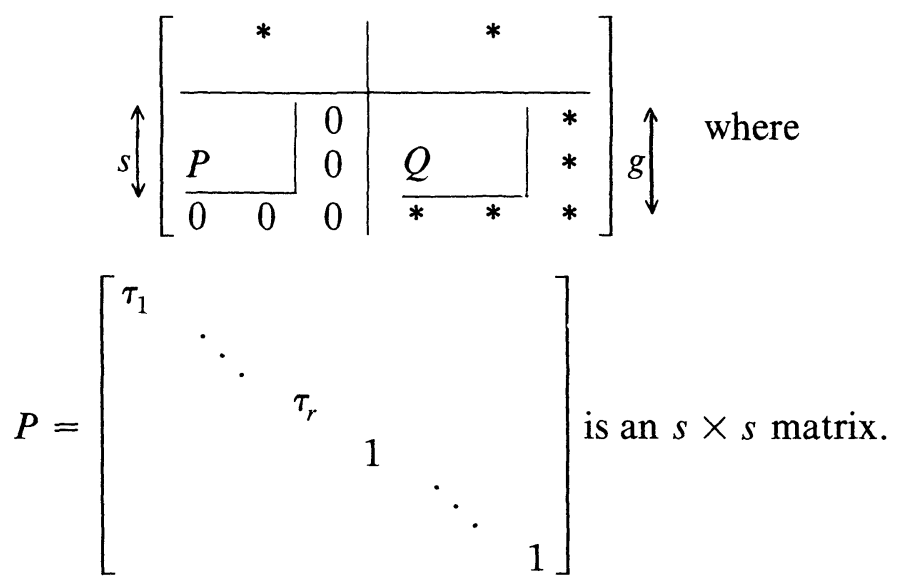

Birman's invariant is det $Q \bmod \tau_{1}$ when $r=s$. The matrix $P^{-1} Q$ can be shown (see [S], for instance) to be a matrix of $\mathscr{L}(\vec{M}, X)$ with respect to the basis $B=\left(\bar{a}_{1}, \ldots, \bar{a}_{s}\right)$ of $T(X)$. In our notation,

$A(X, B)=P^{-1} Q=\left[\begin{array}{ccc}\frac{1}{\tau_{1}} & & \\ & \ddots & \\ & & \frac{1}{\tau_{r}}\end{array}\right] Q$, and $Q$ coincides with $\hat{A}(X, B)$.

It is well known that an automorphism of $H_{1}\left(\partial X_{g} ; \mathbf{Z}\right)$ belongs to $\mathscr{M}_{g_{*}}$ if and only if it preserves the intersection form on $H_{1}\left(\partial X_{g} ; \mathbf{Z}\right)$. In other words, $\mathscr{M}_{g_{*}}$ is isomorphic to the symplectic group $S_{p}^{+}(2 g, \mathbf{Z})$. The subgroup $\mathscr{T}_{g_{*}}{ }^{g_{*}}$ is completely described in Lemma 2.2 of [Bi1]. In Lemma 6.1 we restate Birman's characterisation of $\mathscr{T}_{g_{*}}$ in our notation.

6.1. Lemma. The element $\sigma \in \mathscr{M}_{g_{*}}$ belongs to $\mathscr{T}_{g_{*}}$ if and only if $\sigma(\operatorname{Ker} \varepsilon)=\operatorname{Ker} \varepsilon$ 
Proof. See Lemma 2.2. of [Bi1].

6.2. Lemma. Given $\phi_{*}, \psi_{*} \in \mathscr{M}_{g_{*}}$, they belong to the same double coset modulo $\mathscr{T}_{g_{*}}$ if and only if there exists $\sigma \in \mathscr{T}_{g_{*}}$ such that

$$
\sigma \psi_{*}(\operatorname{Ker} \varepsilon)=\phi_{*}(\operatorname{Ker} \varepsilon) \text {. }
$$

Proof. $\phi_{*}^{-1} \sigma \psi_{*} \in \mathscr{T}_{g_{*}}$ for some $\sigma \in \mathscr{T}_{g_{*}}$ if and only if

$$
\begin{aligned}
\phi_{*}^{-1} \sigma \psi_{*}(\operatorname{Ker} \varepsilon) & =\operatorname{Ker} \varepsilon, \\
\sigma \psi_{*}(\operatorname{Ker} \varepsilon) & =\phi_{*}(\operatorname{Ker} \varepsilon) .
\end{aligned}
$$

7. The linking form of $\vec{M}$ lifted to $\partial X$. The aim of this section is to introduce a new bilinear form associated to an oriented Heegaard splitting $(\vec{M}, X)$. It is defined on a subgroup of $H_{1}(\partial X)$ and takes values in the field of the rationals, $\mathbf{Q}$. It does not provide any other invariant of $(\vec{M}, X)$ more than $\mathscr{L}(\vec{M}, X)$, but will allow us to prove in the next section that the congruence class of $\mathscr{L}(\vec{M}, X)$ determines the double coset associated to $(\vec{M}, X)$ in $\mathscr{M}_{g_{*}}$.

Let $T(\partial X)$ be the preimage of $T(M)$ under the homomorphism

$$
(j \mid)_{*}: H_{1}(\partial X ; \mathbf{Z}) \rightarrow H_{1}(M ; \mathbf{Z})
$$

induced by the inclusion $j: X \hookrightarrow M$.

Given $x, y \in T(\partial X)$, represented by 1 -cycles $\alpha$ and $\beta$, respectively, there exist integers $m, n$ such that $m \alpha=\partial A, n \beta=\partial B$ for some 2-chains $A, B$ in $M$. Let us define

$$
\mathscr{C}(\vec{M}, X)(x, y)=\operatorname{lk}\left(\alpha^{+}, \beta\right)
$$

where $\alpha^{+}$denotes the cycle $\alpha$ pushed out of $X$, and lk denotes the linking as defined in [Se-T], p. 288, i.e.

$$
\operatorname{lk}\left(\alpha^{+}, \beta\right)=\frac{1}{m} A^{+} \cdot \beta=\frac{1}{m} \alpha^{+} \cdot B
$$

(where the dot denotes algebraic intersection in $M$, and $A^{+}$is again the chain $A$ pushed out of $X$ ).

Then,

$$
\mathscr{C}(\vec{M}, X): T(\partial X) \times T(\partial X) \rightarrow \mathbf{Q}
$$

defines a non-symmetric bilinear form. For if $\alpha-\alpha^{\prime}=\partial C$, where $C$ is a 2-chain on $F$, then $\alpha^{+}-\alpha^{++}=\partial C^{+}$and $C^{+} \cdot \beta=0$. Analogously, if $\beta-\beta^{\prime}=\partial D$, where $D$ is a 2 -chain on $F, \alpha^{+} \cdot D=0$. Thus, $\mathscr{C}(\vec{M}, X)$ is well defined and is bilinear. Finally, the property:

$$
\mathscr{C}(\vec{M}, X)(x, y)-\mathscr{C}(\vec{M}, X)(y, x)=x \cdot y
$$


(where the dot denotes the algebraic intersection on $\partial X$ ) generalizes the corresponding property satisfied by the Seifert form on orientable spanning surfaces of links in $S^{3}$, and is easily proven the same way.

The pairing defined above could be defined for any orientable surface (with or without boundary) embedded in a 3-manifold. In his thesis, D. Cooper defined hermitian forms based on these pairings and used their signatures to get invariant of knot and link cobordism [Co]. However for Heegaard surfaces these signatures always vanish.

Next we prove some basic lemmas about $\mathscr{C}(\vec{M}, X)$ and its relation with $\mathscr{L}(\vec{M}, X)$.

Let

$$
\begin{aligned}
i_{X}: H_{1}(\partial X ; \mathbf{Z}) & \rightarrow H_{1}(X ; \mathbf{Z}) \text { and } \\
i_{M-X}: H_{1}(\partial X ; \mathbf{Z}) & \rightarrow H_{1}(\overline{M-X} ; \mathbf{Z})
\end{aligned}
$$

denote inclusion induced homomorphisms.

Note that $T(\partial X)$ is a direct summand of $H_{1}(\partial X)$ and there are symplectic bases $\left(c_{1}, \ldots, c_{g}, a_{1}, \ldots, a_{g}\right)\left(c_{l} \cdot c_{j}=0, a_{i} \cdot a_{j}=0, c_{i} \cdot a_{j}=\right.$ $\left.\delta_{i j}\right)$ such that $\left(c_{1}, \ldots, c_{g}\right)$ is a basis for $\operatorname{Ker} i_{X}$ and $\left(c_{1}, \ldots, c_{g}, a_{1}, \ldots, a_{s}\right)$ is a basis for $T(\partial X)$. Similarly, there exist bases $\left(d_{1}, \ldots, d_{g}, b_{1}, \ldots, b_{g}\right)$ such that $d_{i} \cdot d_{j}=0, b_{i} \cdot b_{j}=0, d_{i} \cdot b_{j}=-\delta_{i j}$ for $1 \leq i, j \leq g,\left(d_{1}, \ldots, d_{g}\right)$ is a basis of $\operatorname{Ker} i_{M-X}$ and $\left(d_{1}, \ldots, d_{g}, b_{1}, \ldots, b_{s}\right)$ is a basis of $T(\partial X)$. (The integer $g-s$ is the first Betti number of $H_{1}(M)$.)

7.1. LEMMA. (i) The matrix of $\mathscr{C}(\vec{M}, X)$ with respect to the basis $\left(c_{1}, \ldots, c_{g}, a_{1}, \ldots, a_{s}\right)$ has the form

$$
\left[\begin{array}{ll}
0 & E \\
0 & C
\end{array}\right]
$$

(ii) The matrix of $\mathscr{C}(\vec{M}, X)$ with respect to the basis $\left(d_{1}, \ldots, d_{g}\right.$, $b_{1}, \ldots, b_{s}$ ) has the form

$$
\left[\begin{array}{ll}
0 & 0 \\
F & D
\end{array}\right]
$$

where $C$ and $D$ are $s \times s$ symmetric matrices with rational entries, $E=\left[\begin{array}{c}I_{s} \\ 0\end{array}\right]$ is a $g \times s$ matrix, $F=\left[I_{s} 0\right]$ is an $s \times g$ matrix and $I_{s}$ is the $s \times s$ identity matrix.

The classes $\{C\}$ and $\{D\}$ of matrices over $\mathbf{Q} / \mathbf{Z}$ are coordinate matrices for $\mathscr{L}(\vec{M}, X)$.

Proof. It is straightforward using the property (7)

$$
\mathscr{C}(\vec{M}, X)(x, y)-\mathscr{C}(\vec{M}, X)(y, x)=x \cdot y .
$$


7.2. LEMMA. Let $(\vec{M}, X),\left(\vec{M}^{\prime}, X^{\prime}\right)$ be Heegaard splittings and

$$
h: H_{1}(\partial X) \rightarrow H_{1}\left(\partial X^{\prime}\right)
$$

be an isomorphism such that $h T(\partial X)=T\left(\partial X^{\prime}\right)$. The following properties are equivalent:

(1) $\mathscr{C}^{\prime}(h x, h y)=\mathscr{C}(x, y)$ for every $x, y \in T(\partial X)$, where $\mathscr{C}, \mathscr{C}^{\prime}$ denote $\mathscr{C}(\vec{M}, X), \mathscr{C}\left(\vec{M}^{\prime}, X^{\prime}\right)$, respectively.

(2) The coordinate matrix of $h$ with respect to the bases $\left(c_{1}, \ldots, c_{g}\right.$, $\left.a_{1}, \ldots, a_{g}\right)$ and $\left(c_{1}^{\prime}, \ldots, c_{g}^{\prime}, a_{1}^{\prime}, \ldots, a_{g}^{\prime}\right)$ is an (integer) matrix of the form

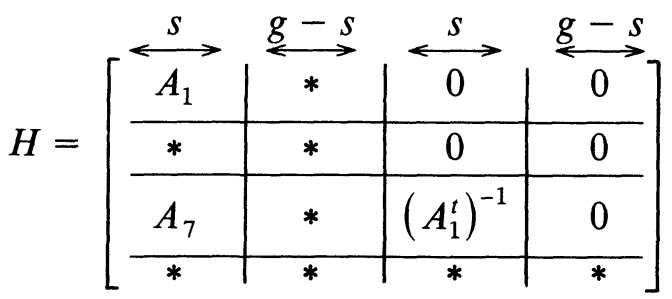

where $A_{1} \in \mathrm{GL}(s, \mathbf{Z})$ and $A_{7}=C A_{1}-\left(A_{1}^{t}\right)^{-1} C^{\prime}$

(3) The coordinate matrix of $h$ with respect to the bases

$$
\left(d_{1}, \ldots, d_{g}, b_{1}, \ldots, b_{g}\right) \text { and }\left(d_{1}^{\prime}, \ldots, d_{g}^{\prime}, b_{1}^{\prime}, \ldots, b_{g}^{\prime}\right)
$$

has the form

$\left[\begin{array}{c|c|c|c}\stackrel{s}{\leftrightarrow} & \stackrel{g-s}{\leftrightarrow} & \stackrel{s}{\longrightarrow} & \stackrel{g-s}{\longrightarrow} \\ \hline 0 & * & 0 & 0 \\ \hline B_{7} & * & \left(B_{1}^{t}\right)^{-1} & 0 \\ \hline * & * & * & *\end{array}\right]$

where $B_{1} \in \mathrm{GL}(s, \mathbf{Z})$ and $B_{7}=D B_{1}-\left(B_{1}^{t}\right)^{-1} D^{\prime}$ and where the stars denote submatrices of $H$ and $G$, respectively.

Proof. Let

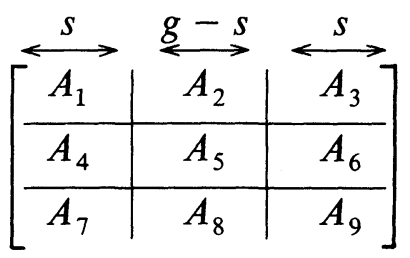

be the submatrix of $H$ consisting on its first $g+s$ rows and columns. Condition (1) is equivalent to

$$
\left[\begin{array}{c|c|c}
A_{1} & A_{2} & A_{3} \\
\hline A_{4} & A_{5} & A_{6} \\
\hline A_{7} & A_{8} & A_{9}
\end{array}\right]\left[\begin{array}{l|c}
0 & I_{s} \\
\cline { 2 - 4 } & 0 \\
\hline 0 & C^{\prime}
\end{array}\right]\left[\begin{array}{c|c|c}
A_{1}^{t} & A_{4}^{t} & A_{7}^{t} \\
\hline A_{2}^{t} & A_{5}^{t} & A_{8}^{t} \\
\hline A_{3}^{t} & A_{6}^{t} & A_{9}^{t}
\end{array}\right]=\left[\begin{array}{c|c}
0 & I_{s} \\
\hline 0 & 0 \\
\hline 0 & C^{\prime}
\end{array}\right]
$$


which gives the equations

$$
\begin{aligned}
& \left(A_{1}+A_{3} C^{\prime}\right) A_{3}^{t}=0, \\
& \left(A_{1}+A_{3} C^{\prime}\right) A_{6}^{t}=0, \\
& \left(A_{1}+A_{3} C^{\prime}\right) A_{9}^{t}=I_{s}, \\
& A_{6} C^{\prime} A_{3}^{t}=0, \\
& A_{6} C^{\prime} A_{6}^{t}=0, \\
& A_{6} C^{\prime} A_{9}^{t}=0, \\
& \left(A_{7}+A_{9} C^{\prime}\right) A_{3}^{t}=0, \\
& \left(A_{7}+A_{9} C^{\prime}\right) A_{6}^{t}=0, \\
& \left(A_{7}+A_{9} C^{\prime}\right) A_{9}^{t}=C .
\end{aligned}
$$

From (3), it follows that $\left(A_{1}+A_{3} C^{\prime}\right) \in \mathrm{GL}(s, \mathbf{Q})$, which together with (1) and (2) implies that $A_{3}=A_{6}=0$. Thus, (4), (5), (6), (7) and (8) are redundant, and $A_{1} A_{9}^{t}=I_{s}$. Equation (9) tell us that

$$
A_{7}=C\left(A_{9}^{t}\right)^{-1}-A_{9} C^{\prime}=C A_{1}-\left(A_{1}^{t}\right)^{-1} C^{\prime} .
$$

The other zero blocks appear since $h T(\partial X)=T\left(\partial X^{\prime}\right)$. The form of $G$ is obtained the same way.

We say that $\mathscr{C}(\vec{M}, X)$ and $\mathscr{C}\left(\vec{M}^{\prime}, X^{\prime}\right)$ are congruent if there exist $h$ as in Lemma 7.2, (1).

7.3. Corollary. If $\mathscr{C}(\vec{M}, X)$ and $\mathscr{C}\left(\vec{M}^{\prime}, X^{\prime}\right)$ are congruent by

$$
h: H_{1}(\partial X) \rightarrow H_{1}\left(\partial X^{\prime}\right)
$$

such that $h T(\partial X)=T\left(\partial X^{\prime}\right)$, then $h$ maps the subgroups $\operatorname{Ker} i_{X}$ and $\operatorname{Ker} i_{M-X}$ to $\operatorname{Ker} i_{X^{\prime}}$ and $\operatorname{Ker}_{M^{\prime}-X^{\prime}}$, respectively.

Proof. By Lemma 7.2(2) $h$ maps Ker $i_{X}=\left\langle c_{1}, \ldots, c_{g}\right\rangle$ to Ker $i_{X^{\prime}}=$ $\left\langle c_{1}^{\prime}, \ldots, c_{g}^{\prime}\right\rangle$ and by Lemma 7.3(3) it sends $\operatorname{Ker} i_{M-X}=\left\langle d_{1}, \ldots, d_{g}\right\rangle$ to $\operatorname{Ker} i_{M^{\prime}-X^{\prime}}=\left\langle d_{1}^{\prime}, \ldots, d_{g}^{\prime}\right\rangle$.

7.4. Corollary. $\mathscr{C}(\vec{M}, X)$ and $\mathscr{C}\left(\vec{M}^{\prime}, X^{\prime}\right)$ are congruent if and only if $\mathscr{L}(\vec{M}, X)$ and $\mathscr{L}\left(\vec{M}^{\prime}, X^{\prime}\right)$ are.

7.5. REMARK. If $\theta$ denotes the intersection pairing on $H_{1}(\partial X)$, it follows from the equation $\mathscr{C}(\vec{M}, X)-\mathscr{C}(\vec{M}, \overrightarrow{M-X})=\theta$ and from Corollary 7.4 that $\mathscr{L}(\vec{M}, X)$ and $\mathscr{L}\left(\vec{M}^{\prime}, X^{\prime}\right)$ are congruent if and only if $\mathscr{L}(\vec{M}, \overline{M-X})$ and $\mathscr{L}\left(\vec{M}^{\prime}, M^{\prime}-X^{\prime}\right)$ are. 
8. Linking form and double cosets. In this section we prove the following

8.1. TheOrem. Let $\phi, \phi^{\prime} \in \mathscr{M}_{\mathrm{g}}$ define oriented Heegaard splittings $(\vec{M}, X)$ and $\left(\vec{M}^{\prime}, X^{\prime}\right)$. Then $\mathscr{L}(\vec{M}, X)$ and $\mathscr{L}\left(\vec{M}^{\prime}, X^{\prime}\right)$ are congruent if and only if $\phi_{*}$ and $\phi_{*}^{\prime}$ belong to the same double coset in $\mathscr{M}_{g_{*}} \bmod \mathscr{T}_{g_{*}}$.

Proof. Assume that $\mathscr{L}(\vec{M}, X)$ and $\mathscr{L}\left(\vec{M}^{\prime}, X^{\prime}\right)$ are congruent. We want to construct an isomorphism $\sigma$ of $H_{1}\left(\partial X_{g}\right)$ such that $\sigma \in \mathscr{T}_{g_{*}}$ and $\sigma \phi_{*}(\operatorname{Ker} \varepsilon)=\phi_{*}^{\prime}(\operatorname{Ker} \varepsilon)$. Then, by Lemma 6.2 we will have proved that $\phi_{*}$, $\phi_{*}^{\prime}$ belong to the same double coset in $\mathscr{M}_{g_{*}} \bmod \mathscr{T}_{g_{*}}$.

Let $u, v \tau: X_{g} \rightarrow M$ be the embeddings of $X_{g}$ onto $X, \overline{M-X}$ respectively. Denote $\bar{u}, \bar{v} \tau$ the homeomorphisms from $\partial X_{g}$ to $\partial X$ induced by the embeddings.

Let $\left(a_{1}, \ldots, a_{s}\right)$ be a basis for $T(X)$ and let $C$ be a symmetric $s \times s$ matrix with rational entries whose class $\{C\}$ in $M(s, \mathbf{Q} / \mathbf{Z})$ is a matrix for $\mathscr{L}(\vec{M}, X)$ with respect to the basis $\left(a_{1}, \ldots, a_{s}\right)$. Denote with primes the corresponding concepts associated to $\left(\vec{M}^{\prime}, X^{\prime}\right)$. Since $\mathscr{L}(\vec{M}, X)$ and $\mathscr{L}\left(\vec{M}^{\prime}, X^{\prime}\right)$ are congruent, there exists a matrix $A \in \operatorname{GL}(s, \mathbf{Z})$ such that

$$
A C^{\prime} A^{t}-C
$$

is an integer matrix. The rows of $A$ are the coordinates of the image of $a_{1}, \ldots, a_{s}$ under an isomorphism $h: T(X) \rightarrow T\left(X^{\prime}\right)$ with respect to the basis $\left(a_{1}^{\prime}, \ldots, a_{s}^{\prime}\right)$.

Since $H_{1}(X) / T(X)$ is isomorphic to $H_{1}(M) / T(M)$ and this is a free abelian group, it follows that $T(X)$ is a direct summand of $H_{1}(X)$. Therefore $\left(a_{1}, \ldots, a_{s}\right)$ can be extended to a basis $\left(a_{1}, \ldots, a_{s}, a_{s+1}, \ldots, a_{g}\right)$ of $H_{1}(X)$. The same is true for $\left(\vec{M}^{\prime}, X^{\prime}\right)$. We extend $h$ to an isomorphism ext $h: H_{1}(X) \rightarrow H_{1}\left(X^{\prime}\right)$ by defining

$$
\operatorname{ext} h\left(a_{i}\right)=a_{i}^{\prime}, \quad s<i \leq g .
$$

Let $0, I \in M(g-s, \mathbf{Z})$ denote the zero and identity elements, respectively. The coordinate matrix of ext $h$ with respect to the bases $\left(a_{1}, \ldots, a_{g}\right)$, $\left(a_{1}^{\prime}, \ldots, a_{g}^{\prime}\right)$ is

$$
\bar{A}=A \oplus I
$$

Consider the matrices

$$
\begin{aligned}
\bar{C} & :=C \oplus 0, \\
\bar{C}^{\prime} & :=C^{\prime} \oplus 0 .
\end{aligned}
$$

Then, of course $\bar{A} \bar{C}^{\prime} \bar{A}^{t}-\bar{C}$ is an integer matrix. 
Now the bases $\left(a_{1}, \ldots, a_{g}\right)$ and $\left(a_{1}^{\prime}, \ldots, a_{g}^{\prime}\right)$ lift to symplectic bases $\left(c_{1}, \ldots, c_{g}, a_{1}, \ldots, a_{g}\right)$ and $\left(c_{1}^{\prime}, \ldots, c_{g}^{\prime}, a_{1}^{\prime}, \ldots, a_{g}^{\prime}\right)$ of $H_{1}(\partial X)$ and $H_{1}\left(\partial X^{\prime}\right)$, respectively, where $\left(c_{1}, \ldots, c_{g}\right)$ is a basis of $\operatorname{Ker} i_{X}$ and $\left(c_{1}^{\prime}, \ldots, c_{g}^{\prime}\right)$ is a basis of $\operatorname{Ker} i_{X^{\prime}}$

The (symplectic) matrix

$$
\left[\begin{array}{ll}
\left(\overline{A^{t}}\right)^{-1} & 0 \\
-\bar{A} \bar{C}^{\prime}+\bar{C}\left(\overline{A^{t}}\right)^{-1} & \bar{A}
\end{array}\right]
$$

is the coordinate matrix with respect to the bases $\left(c_{1}, \ldots, c_{g}, a_{1}, \ldots, a_{g}\right)$ and $\left(c_{1}^{\prime}, \ldots, c_{g}^{\prime}, a_{1}^{\prime}, \ldots, a_{g}^{\prime}\right)$ of an isomorphism $\bar{\sigma}$ from $H_{1}(\partial X)$ to $H_{1}\left(\partial X^{\prime}\right)$. Therefore $\bar{\sigma}$ takes $\operatorname{Ker} i_{X}$ to $\operatorname{Ker} i_{X^{\prime}}$. We can assume that the matrices $C$, $C^{\prime}$ are blocks of matrices of the pairings $\mathscr{C}(\vec{M}, X)$ and $\mathscr{C}\left(\vec{M}^{\prime}, X^{\prime}\right)$ as in Lemma 7.1. Since the matrix (8) has the form $H$ in Lemma 7.2.(2) it follows that $\bar{\sigma}$ preserves these pairings. Then, by Corollary 7.3, $\bar{\sigma}$ takes $\operatorname{Ker} i_{M-X}$ to $\operatorname{Ker} i_{M^{\prime}-X^{\prime}}$

Define $\sigma:=\left.\left(u_{*}^{\prime}\right)^{-1} \bar{\sigma} u\right|_{*}$.

Recall that $\phi=\left.\left.u^{-1} v\right|_{,} u\right|_{*} \operatorname{Ker} \varepsilon=\operatorname{Ker} i_{X}$ and $\left.v\right|_{*} \operatorname{Ker} \varepsilon=\operatorname{Ker} i_{M-X}$. The analogous holds for $\left(\vec{M}^{\prime}, X^{\prime}\right)$. Finally,

$$
\begin{gathered}
\sigma(\operatorname{Ker} \varepsilon)=u_{*}^{\prime-1} \bar{\sigma}\left(\operatorname{Ker} i_{X}\right)=u_{*}^{\prime-1}\left(\operatorname{Ker} i_{X^{\prime}}\right)=\operatorname{Ker} \varepsilon \\
\sigma\left(\phi_{*} \operatorname{Ker} \varepsilon\right)=\left.u_{*}^{\prime-1} \bar{\sigma} u\right|_{*}\left(\left.u\right|_{*} ^{-1} \operatorname{Ker} i_{M-X}\right) \\
=u_{*}^{\prime-1}\left(\operatorname{Ker} i_{M^{\prime}-X^{\prime}}\right)=\phi_{*}^{\prime}(\operatorname{Ker} \varepsilon),
\end{gathered}
$$

Thus by Lemmas 6.1 and 6.2 it follows that $\phi_{*}$ and $\phi_{*}^{\prime}$ belong to the same double coset in $\mathscr{M}_{g_{*}}$ modulo $\mathscr{T}_{g_{*}}$.

8.2. REMARK. As a consequence of Theorem 8.1 we see that for any two $\phi, \phi^{\prime} \in \mathscr{M}_{g}$ defining Heegaard splittings of $M, M^{\prime}$ such that $H_{1}(M)$ $\cong H_{1}\left(M^{\prime}\right)$ and $g>\operatorname{rank} H_{1}(M)+r$, where $r$ is the number of torsion coefficients of $T(M)$, then $\phi_{*}$ and $\phi_{*}^{\prime}$ belong to the same double coset modulo $\mathscr{T}_{g_{*}}$. This is the case, for instance, when $M, M^{\prime}$ are homological spheres, $H_{1}(M) \cong H_{1}\left(M^{\prime}\right)$ are torsion free or the Heegaard splitting has not minimal genus.

8.3. REMARK. It follows from the theorem that any invariant of the double coset $\mathscr{T}_{g_{*}} \phi_{*} \mathscr{T}_{g_{*}}$ are invariants of the congruence class of $\mathscr{L}(\vec{M}, X)$ and vice versa. Since we have given a complete invariant of $\mathscr{L}(\vec{M}, X)$, we have determined a complete invariant of the double coset.

9. Applications: connected sums of lens spaces. In practice we want to classify unoriented Heegaard splittings $(M, F)$ as defined in $\S 1$. An unoriented Heegaard splitting defines four oriented ones, namely $(\vec{M}, X)$, 
$(\vec{M}, Y),(\overleftarrow{M}, X),(\overleftarrow{M}, Y)$ where $X, Y$ are the handlebodies separated by $F$. Therefore a homeomorphism $f:(M, F) \rightarrow\left(M^{\prime}, F^{\prime}\right)$ must send each one of the unordered pairs $\{(\vec{M}, X),(\vec{M}, Y)\},\{(\overleftarrow{M}, X),(\overleftarrow{M}, Y)\}$, to one of the unordered pairs $\left\{\left(\vec{M}^{\prime}, X^{\prime}\right) ;\left(\vec{M}^{\prime}, Y^{\prime}\right)\right\},\left\{\left(\overleftarrow{M}^{\prime}, X^{\prime}\right),\left(\overleftarrow{M}^{\prime}, Y^{\prime}\right)\right\}$. Therefore the unordered pairs

$$
\{(\vec{M}, X),(\vec{M}, Y)\}, \quad\{(\overleftarrow{M}, X),(\overleftarrow{M}, Y)\}
$$

form an invariant of $(M, F)$. In this way we can use the invariants defined before to distinguish $(M, F)$ among unoriented Heegaard splittings.

In this section we apply our invariants to obtain some results on the classification of Heegaard splittings of connected sums of lens spaces. We take, for simplicity, the connected sum of two lens spaces.

Let $p, s$ be positive integers with $\operatorname{gcd}(p, s)=1$. The lens space $\vec{L}(p, s)$ is obtained by $-p / s$ Dehn-surgery on $\vec{S}^{3}$ along the trivial knot. The boundary of a tubular neighborhood of the trivial knot is a torus $F$ which gives a Heegaard splitting of $L(p, s)$ of genus 1 . We denote by $X$ and $Y$ the solid tori separated by $F$, where $Y$ contains the core of the surgery.

Consider a canonical meridian-longitude pair $(m, l)$ on $F$, oriented so that $m \cdot l=+1$ on $\partial \vec{Y}$. Then $x=m$ and $y=-\hat{s} m+\hat{p} l$ are bases for $H_{1}(X)$ and $H_{1}(Y)$, respectively, where $p \hat{p}-s \hat{s}=1$. Moreover, $j_{*}(x)$ and $j_{*}(y)$ both generate $H_{1}(L(p, s))$, which is isomorphic to $(p)$. Thus $T(X)=H_{1}(X)$ and $T(Y)=H_{1}(Y)$. It is not difficult to check that $\mathscr{L}(x, x)=s / p$. Therefore, $\mathscr{L}(y, y)=\mathscr{L}(-\hat{s} m+\hat{p} l,-\hat{s} m+\hat{p} l)=$ $\mathscr{L}(-\hat{s} m,-\hat{s} m)=\left(\hat{s}^{2} s / p\right)=\hat{s} / p$. Thus

$$
A(X, x)=\left[\frac{s}{p}\right] \text { and } A(Y, y)=\left[\frac{\hat{s}}{p}\right]
$$

and the unordered pairs $\pm\{s \bmod p ; \hat{s} \bmod p\}$ form an invariant of the equivalence class of $(L(p, s), F)$.

Thus if $(L(p, s), F)$ is equivalent to $\left(L\left(p, s^{\prime}\right), F^{\prime}\right)$ one of the four conditions hold:

(i) $(\vec{L}(p, s), X) \cong\left(\vec{L}\left(p, s^{\prime}\right), X\right) \Rightarrow s \equiv s^{\prime} \bmod p$,

(ii) $(\vec{L}(p, s), X) \cong\left(\vec{L}\left(p, s^{\prime}\right), Y\right) \Rightarrow s \equiv s^{\prime} \bmod p \Leftrightarrow s s^{\prime} \equiv 1 \bmod p$,

(iii) $(\vec{L}(p, s), X) \cong\left(\overleftarrow{L}\left(\underline{p}, s^{\prime}\right), X\right) \Rightarrow s \equiv-s^{\prime} \bmod p$,

(iv) $(\vec{L}(p, s), X) \cong\left(\stackrel{L}{L}\left(p, s^{\prime}\right), Y\right) \Rightarrow s \equiv-\hat{s}^{\prime} \bmod p \Leftrightarrow s s^{\prime} \equiv-1$ $\bmod p$.

In each case, the reciprocal assertion is also true. If the conditions on $s, s^{\prime}$ are satisfied, it is easy to construct homeomorphisms between the pairs of oriented manifolds. Note that in this way the topological classification of lens spaces, which was obtained by Brody follows by virtue of 
the Bonahon-Otal Theorem [Bo-O] or by Hodgon's M.Sc. Thesis [H], as it is well known.

The above classification of Heegaard splittings of lens spaces is in [Bi1]. We add it here since we want to use it when $s=s^{\prime}$. If $s^{2} \equiv 1 \bmod p$ then $(\vec{L}(p, s), X) \cong(\vec{L}(p, s), Y)$ and we call this Heegaard splitting invertible (by analogy with knot theory).

We now study the connected sum of two lens spaces. Given two oriented Heegaard splittings $\left(\vec{M}_{1}, X_{1}\right)$ and $\left(\vec{M}_{2}, X_{2}\right)$ the pair $\left(\vec{M}_{1} \# \vec{M}_{2}\right.$, $\left.X_{1} \mapsto X_{2}\right)$ is well defined [Wa] and it is an oriented Heegaard splitting.

According to a theorem of Haken, any Heegaard splitting of a connected sum of manifolds $\vec{M}_{1} \# \vec{M}_{2}$ is equivalent to a connected sum of a Heegaard splitting of $M_{1}$ and a Heegaard splitting of $M_{2}$. On the other hand, Bonahon-Otal [Bo-O] proved that any two Heegaard splittings of genus $g>1$ of a lens space $L(p, s)$ are ambient isotopic, and in particular are ambient isotopic to the stabilization of the canonical Heegaard splitting of genus one (for lens spaces non-homeomorphic to $S^{3}$ ). Therefore, it follows that the Heegaard genus of the connected sum $\vec{L}(p, s) \#$ $\vec{L}\left(p^{\prime}, s^{\prime}\right)$ of two lens spaces is two, and that any Heegaard splitting of genus two is equivalent to a connected sum of the canonical Heegaard splittings of the lens $L(p, s), L\left(p^{\prime}, s^{\prime}\right)$ respectively. Thus, any unoriented Heegaard splitting of genus two of $\vec{L}(p, s) \# \vec{L}\left(p^{\prime}, s^{\prime}\right)$ is equivalent either to $\left(\vec{L}(p, s) \# \vec{L}\left(p^{\prime}, s^{\prime}\right), \partial\left(X \boxminus X^{\prime}\right)\right)$ or to $\left(\vec{L}(p, s) \# \vec{L}\left(p^{\prime}, s^{\prime}\right)\right.$, $\left.\partial\left(X \mapsto Y^{\prime}\right)\right)$. It is known that sometimes these Heegaard splittings are inequivalent [Eng], [Bi1], and that any Heegaard splitting of genus $>2$ of $\vec{L}(p, s) \# \vec{L}\left(p^{\prime}, s^{\prime}\right)$ is isotopic to the stabilization of one of these Heegaard splittings of genus 2.

Consider the bases

$$
\begin{aligned}
& B\left(X \boxminus X^{\prime}\right):=\left(x, x^{\prime}\right) \\
& B\left(Y \boxminus Y^{\prime}\right):=\left(y, y^{\prime}\right)
\end{aligned}
$$

of $T\left(X \boxminus X^{\prime}\right)$ and $T\left(Y \boxminus Y^{\prime}\right)$, respectively, for $\left(\vec{L}(p, s) \# \vec{L}\left(p^{\prime}, s^{\prime}\right), X\right.$ ต $\left.X^{\prime}\right)$. Consider also the bases

$$
\begin{aligned}
& B^{\prime}\left(X \boxminus Y^{\prime}\right):=\left(x, y^{\prime}\right), \\
& B^{\prime}\left(Y \boxminus X^{\prime}\right):=\left(y, x^{\prime}\right)
\end{aligned}
$$

of $T\left(X \boxminus Y^{\prime}\right)$ and $T\left(Y \boxminus X^{\prime}\right)$, respectively, for $\left(\vec{L}(p, s) \# \vec{L}\left(p^{\prime}, s^{\prime}\right), X\right.$ $\natural Y)$. Therefore we have the following matrices:

$$
\begin{aligned}
& \hat{A}\left(X \boxminus X^{\prime}, B\right)=\left[\begin{array}{cc}
s & 0 \\
0 & s^{\prime}
\end{array}\right], \quad \hat{A}\left(Y \text { घ } Y^{\prime}, B\right)=\left[\begin{array}{cc}
\hat{s} & 0 \\
0 & \hat{s}^{\prime}
\end{array}\right], \\
& \hat{A}\left(X \natural^{\prime}, B^{\prime}\right)=\left[\begin{array}{cc}
s & 0 \\
0 & \hat{s}^{\prime}
\end{array}\right], \quad \hat{A}\left(Y \nvdash X^{\prime}, B^{\prime}\right)=\left[\begin{array}{cc}
\hat{s} & 0 \\
0 & s^{\prime}
\end{array}\right] .
\end{aligned}
$$


Let $d$ be the g.c.d. of $p$ and $p^{\prime}$. As a corollary of Theorem 5.1 we have the following.

9.1. Proposition. If

$$
\begin{gathered}
\left(\vec{L}(p, s) \# \vec{L}\left(p^{\prime}, s^{\prime}\right), \partial\left(X \boxminus X^{\prime}\right)\right) \text { and } \\
\left(\vec{L}(p, s) \# \vec{L}\left(p^{\prime}, s^{\prime}\right), \partial\left(X \sharp Y^{\prime}\right)\right)
\end{gathered}
$$

are equivalent, then one of the following holds:
(i) $s^{\prime 2} \equiv 1 \bmod d$,
(ii) $s^{2} \equiv 1 \bmod d$.

EXAMPLE. $\vec{L}(5,2) \# \vec{L}(25,4)$. Here $d=5 . L(25,4)$ satisfies condition (i) and according to Theorem 4.1 the forms $\left[\begin{array}{cc}2 / 5 & 0 \\ 0 & 4 / 25\end{array}\right]$ and $\left[\begin{array}{cc}2 / 5 & 0 \\ 0 & 19 / 25\end{array}\right]$ are congruent (for instance under $C=\left[\begin{array}{cc}-469 & 25 \\ -74 & 4\end{array}\right]$ ). However, most likely the two Heegaard splittings in question are inequivalent.

9.2. Corollary. Under the conditions of Proposition 5.1, if $p=p^{\prime}$, the Heegaard splittings are equivalent if and only if one of the following holds:

(i) $s^{\prime 2} \equiv 1 \bmod p$,

(ii) $s^{2} \equiv 1 \bmod p$.

Proof. If (i) or (ii) holds one of $L(p, s), L\left(p, s^{\prime}\right)$ is invertible.

9.3. Remark. This last result can be also obtained using Birman invariants [Bi] (with the proviso that conditions (32), (33) of [Bi] p. 148 is unnecessary if the genus is even).

9.4. LemMA. Let $\left(M, F_{g}\right),\left(M^{\prime}, F_{h}\right)$ be Heegaard splittings with $g \leq h$. After $g$ stabilizations $\left(\vec{M} \# \vec{M}^{\prime}, \partial\left(X \boxminus X^{\prime}\right)\right)$ and $\left(\vec{M} \# \vec{M}^{\prime}, \partial\left(X \boxminus Y^{\prime}\right)\right)$ become ambient isotopic.

Proof. Let $V\left(F_{g}\right)$ be a tubular neighborhood of $F_{g}$ in $M$. Then $\partial\left(V\left(F_{g}\right)\right) \cong F_{g} \times\{0,1\}$. Denote by $F_{g, 0}$ the surface $F_{g}$ with a hole. Note that $\left(F_{g, 0} \times[0,1]\right)$ provides a Heegaard splitting $\left(M, F_{2 g}\right)$ of genus $2 g$ of $M$, and that $F_{2 g}$ separates the handebodies $X \nvdash Y$ and $F_{g, 0} \times[0,1]$ (as the first author learned from L. Siebenmann). Then $\left(M, F_{2 g}\right)$ is equivalent to $g$ stabilizations of $\left(M, F_{g}\right)$ as can be easily checked directly (see Figure 5.1).

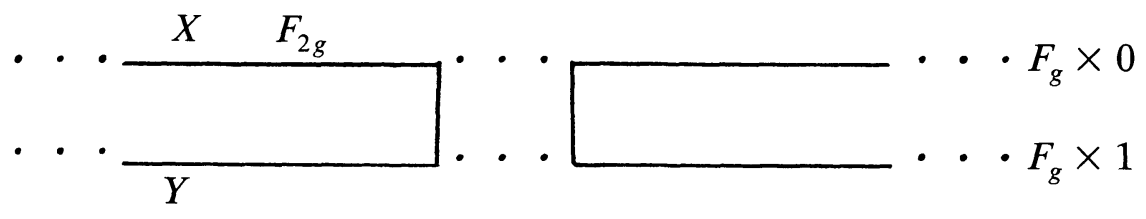

FIGURE 5.1 
Realize the connected sum $\left(\vec{M} \# \vec{M}^{\prime},(X \boxminus Y) \natural X^{\prime}\right)$ using a tubular neighbourhood in $M$ of a disk on $F_{g, 0} \times\{0\}$. Clearly $\left(\vec{M} \# \vec{M}^{\prime}\right.$, $\left.\partial\left((X \curvearrowleft Y) \natural X^{\prime}\right)\right)$ is obtained from $\left(\vec{M} \# \vec{M}^{\prime}, \partial\left(X \boxminus X^{\prime}\right)\right)$ by $g$ stabilizations (Figure 5.2).

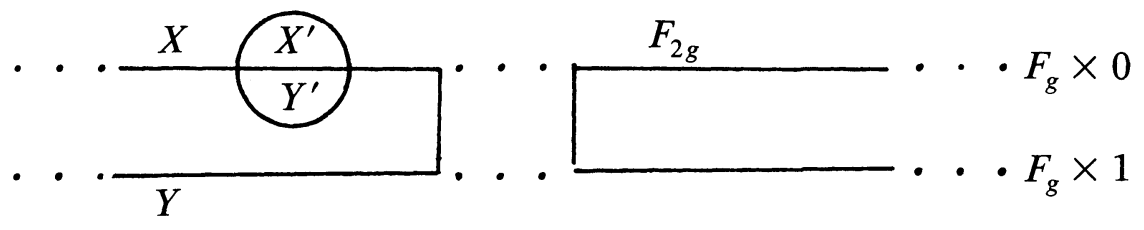

FIGURE 5.2

Analogously, realize the connected sum $\left(\vec{M} \# \vec{M}^{\prime},(X \boxminus Y)\right.$ 曰 $\left.X^{\prime}\right)$ using a tubular neighbourhood in $M$ of a disk on $F_{g, 0} \times\{1\}$ (Figure 5.3). Then $\left(\vec{M} \# \vec{M}^{\prime}, \partial\left((X \boxminus Y) \boxminus X^{\prime}\right)\right)$ is equivalent to $g$ stabilizations in $\left(\vec{M} \# \vec{M}^{\prime}, \partial\left(X \boxminus Y^{\prime}\right)\right)$.

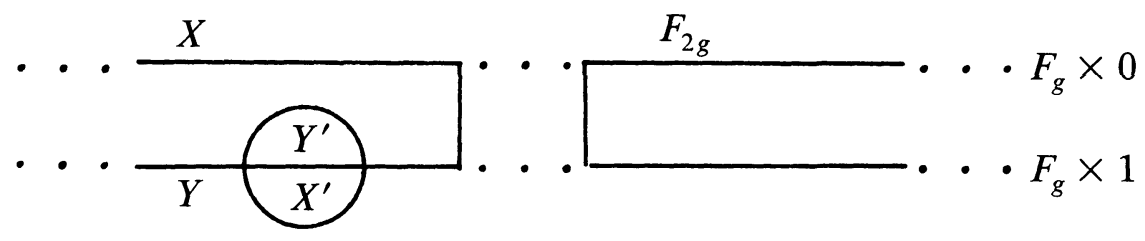

FIGURE 5.3

9.5. Corollary. The Heegaard splittings of genus greater than two of $\vec{L}(p, s) \# \vec{L}\left(p^{\prime}, s^{\prime}\right)$ are equivalent.

9.6. COROLlaRY. (Classification of the Heegaard splittings of $\vec{L}(p, s)$ $\# \vec{L}\left(p, s^{\prime}\right)$.) The Heegaard splittings of genus greater than two of $\vec{L}(p, s)$ \# $\vec{L}\left(p, s^{\prime}\right)$ are equivalent. There is one equivalence class of Heegaard splittings of genus two if $s^{2} \equiv 1$ or $s^{\prime 2} \equiv 1(\bmod p)$, and two equivalence classes otherwise.

\section{REFERENCES}

[Bass] H. Bass, K-theory and stable algebra, Publications Mathematiques IHES no. 22 (1964).

[Bi1] J. S. Birman, On the equivalence of Heegaard splittings of closed orientable 3-manifolds, Ann. Math. Studies, 84 (1975), 137-139.

[Bi2] Lecture notes for the CBMS conference at Blacksburg, Va., 1977.

[Bi-J] J. S. Birman and D. Johnson, Homology Heegaard splittings, manuscript.

[BGAM] J. S. Birman, F. González-Acuña and J. Montesinos, Minimal Heegaard splittings of 3-manifolds are not unique, Michigan Math. J., 23 (1976), 97-103.

[Bi-H] J. S. Birman and H. Hilden, Heegaard splittings of branched coverings of $\mathrm{S}^{3}$, Trans. Amer. Math. Soc., 213 (1975), 315-352. 
[Bo-O] F. Bonahon and S. P. Otal, Scindements de Heegaard des spaces lenticulares, C.

R. Acad. Sci. Paris, 294 (1982), 585-587.

[Bu] E. Burger, Über gruppen mit Verschlingungen, J. Reine Angew. Math., 188 (1950), 193-200.

[Co] D. Cooper, Signatures of surfaces in 3-manfolds and applications to knot and link cobordism, Ph.D., Warwick University (1982).

[Eng] R. Engmann, Nicht homëomorphe Heegaard Zerlegungen von Geslecht 2 der Zusammenhängenden Summe zweier Linsenraüme, Abh. Math. Sem. Univ. Hamburg, 35 (1970), 33-38.

[H] C. Hodgson and J. H. Rubinstein, Involutions and Isotopies of Lens Spaces, Springer LNM \#1144 (1985).

[K] A. Kawauchi and S. Kojima, Algebraic classification of linking pairings on 3-manifolds, Math. Ann., 253 (1980), 29-92.

[Mol] J. Montesinos, Minimal plat representations of prime knots and links are not unique, Canad. J. Math., 28 (1976), 161-167.

[Mo2] J. Montesinos, Revêtements doubles ramifiés de noeuds, varietés de Seifert et diagrams de Heegaard, Polycopié Orsay (1979).

[MS] J. Montesinos and C. Safont, On the Birman invariants of Heegaard splittings, MSRI preprint \#14102-85, November, 1985.

[S] C. Safont, Ph.D. Thesis, University of Zaragoza, 1984.

[Se] H. Seifert, Verschlingungsinvarianten, Sitzumgsberichte der Preuss. Adad. der Wiss., Berlin, (1933), 811-828.

[Se-T] H. Seifert-W. Threlfall, A Textbook of Topology, Academic Press, N. Y. (1980)

[W] C. T. C. Wall, Quadratic forms on finite groups, and related topics, Topology, 2 (1964), 281-298.

[Wa] F. Waldhausen, Heegaard Zerlegungen der 3-sphäre, Topology, 7 (1968), 195-203.

Received October 25, 1985 and in revised form April 9, 1987. The first author was supported by Comité Hispano-Norteamericano, and partially by NSF Grant 8120790 . The second author was supported by MEC-Fullbright grant.

Mathematical Sciences Research Institute, AND

Mathematics DePaRtMENT

COLUMBIA UNIVERSITY

NEW YORK, NY 10027

AND

UNIVERSIDAD DE ZARAGOZA

ZARAGOZA, SPAIN 


\section{PACIFIC JOURNAL OF MATHEMATICS \\ EDITORS}

V. S. VARADARAJAN

(Managing Editor)

University of California

Los Angeles, CA 90024

Herbert Clemens

University of Utah

Salt Lake City, UT 84112

R. FINN

Stanford University

Stanford, CA 94305
HERMANN FLASCHKA

University of Arizona

Tucson, AZ 85721

RAMESH A. GANGOLLI

University of Washington Seattle, WA 98195

VAUGHAN F. R. JONES

University of California

Berkeley, CA 94720
ROBION KIRBY

University of California

Berkeley, CA 94720

C. C. MOORE

University of California

Berkeley, CA 94720

HAROLD STARK

University of California, San Diego

La Jolla, CA 92093

\section{ASSOCIATE EDITORS}
R. ARENS
E. F. BECKENBACH
B. H. NEUMANN
F. WOLF
K. YOSHIDA
(1906-1982)

\section{SUPPORTING INSTITUTIONS}

UNIVERSITY OF ARIZONA

UNIVERSITY OF BRITISH COLUMBIA

CALIFORNIA INSTITUTE OF TECHNOLOGY

UNIVERSITY OF CALIFORNIA

MONTANA STATE UNIVERSITY

UNIVERSITY OF NEVADA, RENO

NEW MEXICO STATE UNIVERSITY

OREGON STATE UNIVERSITY
UNIVERSITY OF OREGON UNIVERSITY OF SOUTHERN CALIFORNIA

STANFORD UNIVERSITY

UNIVERSITY OF HAWAII

UNIVERSITY OF TOKYO

UNIVERSITY OF UTAH

WASHINGTON STATE UNIVERSITY

UNIVERSITY OF WASHINGTON 


\section{Pacific Journal of Mathematics \\ Vol. 132, No. $1 \quad$ January, 1988}

Scott W. Brown, Full analytic subspaces for contractions with rich spectrum ...1

Robert Main Burton, Jr. and Tae-Sung Kim, An invariance principle for

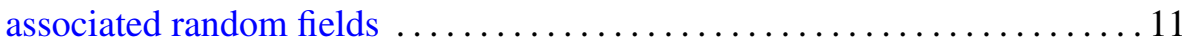

Ana M. Viola-Prioli and Jorge Viola-Prioli, Rings whose kernel functors

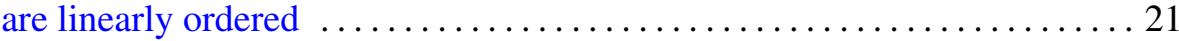

David E. Handelman, Representing polynomials by positive linear

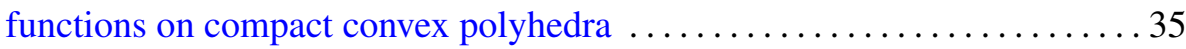

Patrick Keef, On the Tor functor and some classes of abelian groups . .....66

Dennis R. Malm, Simplicity of partial and Schmidt differential operator

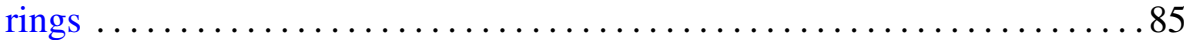

José M. Montesinos and Carmen Safont, On the Birman invariants of

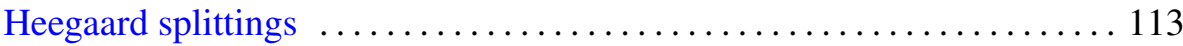

N. P. Mukherjee and Prabir Bhattacharya, The normal index of a finite group

Mario Petrich and Norman R. Reilly, Semigroups generated by certain operators on varieties of completely regular semigroups

Robert Tijdeman and Lian Xiang Wang, Sums of products of powers of given prime numbers

Joel Larry Weiner, First integrals for a direction field on a simply connected plane domain 